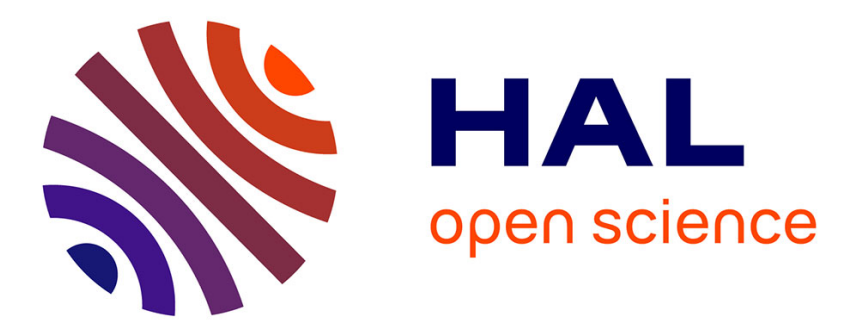

\title{
High Dimensional Single-Cell Analysis Reveals iNKT Cell Developmental Trajectories and Effector Fate Decision
}

Thomas Baranek, Kevin Lebrigand, Carolina de Amat Herbozo, Loïc Gonzalez, Gemma Bogard, Céline Dietrich, Virginie Magnone, Chloé Boisseau, Youenn Jouan, François Trottein, et al.

\section{To cite this version:}

Thomas Baranek, Kevin Lebrigand, Carolina de Amat Herbozo, Loïc Gonzalez, Gemma Bogard, et al.. High Dimensional Single-Cell Analysis Reveals iNKT Cell Developmental Trajectories and Effector Fate Decision. Cell Reports, 2020, 32 (10), pp.108116. 10.1016/j.celrep.2020.108116 . hal-02937420

\author{
HAL Id: hal-02937420 \\ https://hal.science/hal-02937420
}

Submitted on 5 Nov 2020

HAL is a multi-disciplinary open access archive for the deposit and dissemination of scientific research documents, whether they are published or not. The documents may come from teaching and research institutions in France or abroad, or from public or private research centers.
L'archive ouverte pluridisciplinaire $\mathbf{H A L}$, est destinée au dépôt et à la diffusion de documents scientifiques de niveau recherche, publiés ou non, émanant des établissements d'enseignement et de recherche français ou étrangers, des laboratoires publics ou privés. 


\section{Cell Reports}

\section{High Dimensional Single-Cell Analysis Reveals iNKT Cell Developmental Trajectories and Effector Fate Decision}

\section{Graphical Abstract}

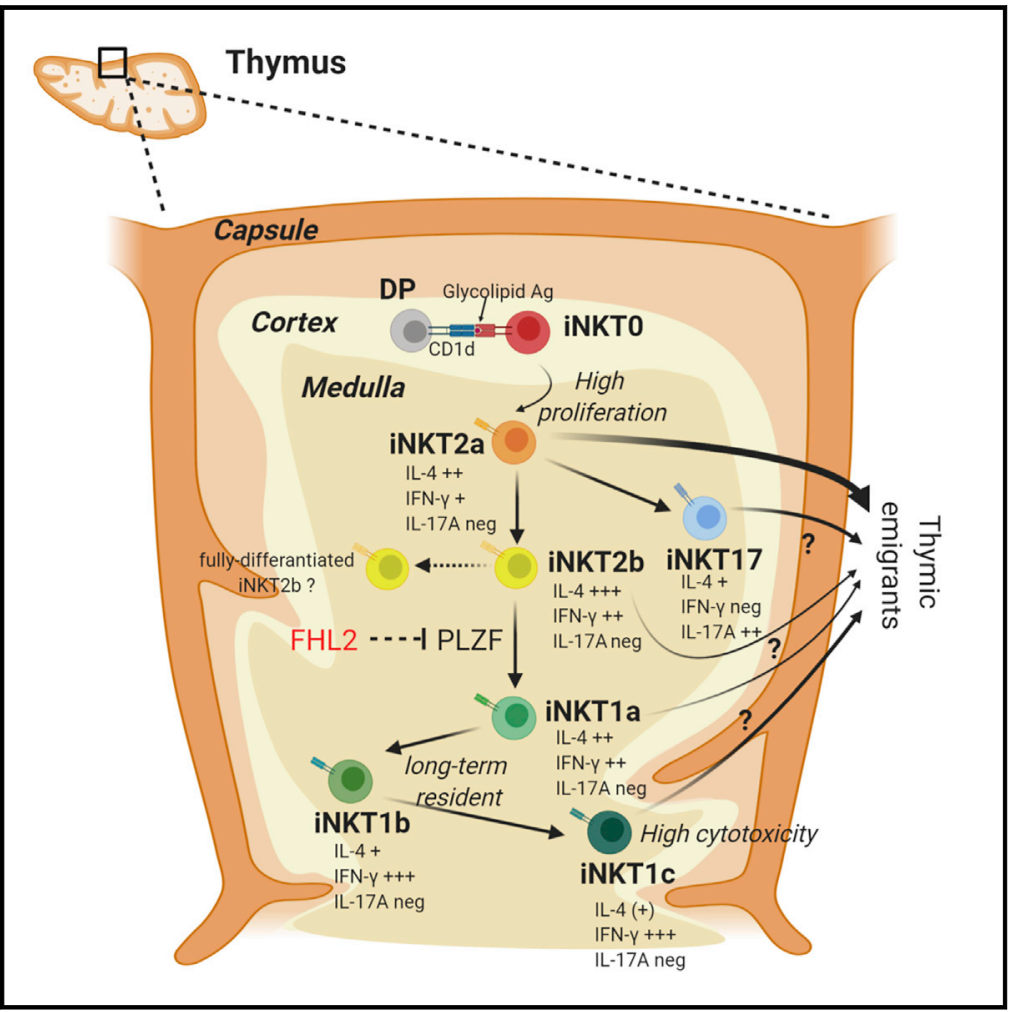

\section{Highlights}

- Unbiased scRNA-seq on thymic iNKT reveals discrete subsets in iNKT1 and iNKT2

- Transcriptomically defined iNKT2 cells comprise precursors to iNKT1 and iNKT17

- Most of thymic iNKT subsets seem to have the potential to egress the thymus

- The transcription cofactor FHL2 controls specification of the iNKT1 effector fate

\section{Authors}

Thomas Baranek, Kevin Lebrigand, Carolina de Amat Herbozo, ..., Maria Leite-de-Moraes, Thierry Mallevaey, Christophe Paget

\section{Correspondence}

baranek@tours-univ.fr (T.B.), christophe.paget@inserm.fr (C.P.)

\section{In Brief}

Baranek et al. perform an unbiased single-cell transcriptomic analysis of thymic iNKT cells, which reveals an unappreciated transcriptional and functional heterogeneity among these innate $T$ cells. Mathematical modelling and biological approaches yield a model that may reconcile the current conflicting models. 
High Dimensional Single-Cell Analysis Reveals iNKT Cell Developmental Trajectories and Effector Fate Decision

\author{
Thomas Baranek, ${ }^{1,2, *}$ Kevin Lebrigand, ${ }^{3}$ Carolina de Amat Herbozo, ${ }^{4}$ Loïc Gonzalez,,$^{1,2}$ Gemma Bogard, ,, 2 \\ Céline Dietrich, ${ }^{5}$ Virginie Magnone, ${ }^{3}$ Chloé Boisseau, ${ }^{1,2}$ Youenn Jouan, ${ }^{1,2,6}$ François Trottein, ${ }^{7}$ Mustapha Si-Tahar, ${ }^{1,2}$ \\ Maria Leite-de-Moraes, ${ }^{5}$ Thierry Mallevaey, ${ }^{4,8}$ and Christophe Paget ${ }^{1,2,9, *}$ \\ ${ }^{1}$ INSERM, Centre d'Etude des Pathologies Respiratoires (CEPR), UMR 1100, Tours, France \\ ¿Université de Tours, Faculté de Médecine de Tours, Tours, France \\ 3Université Côte d'Azur, CNRS, IPMC, Sophia-Antipolis, France \\ ${ }^{4}$ Department of Immunology, University of Toronto, Toronto, ON M5S 1A8, Canada \\ 5 Université de Paris, Paris, France; Laboratory of Immunoregulation and Immunopathology, INEM (Institut Necker-Enfants Malades), CNRS \\ UMR8253 and INSERM UMR1151, Paris, France \\ ${ }^{6}$ Service de Médecine Intensive et Réanimation, Centre Hospitalier Régional Universitaire, Tours, France \\ ${ }^{7}$ Centre d'Infection et d'Immunité de Lille, Inserm U1019, CNRS UMR 9017, University of Lille, CHU Lille- Institut Pasteur de Lille, 59000 Lille, \\ France \\ 8Institute of Biomaterials \& Biomedical Engineering, Toronto, ON M5S 1A8, Canada \\ ILead Contact \\ *Correspondence: baranek@tours-univ.fr (T.B.), christophe.paget@inserm.fr (C.P.) \\ https://doi.org/10.1016/j.celrep.2020.108116
}

\title{
SUMMARY
}

CD1d-restricted invariant Natural Killer T (iNKT) cells represent a unique class of T lymphocytes endowed with potent regulatory and effector immune functions. Although these functions are acquired during thymic ontogeny, the sequence of events that gives rise to discrete effector subsets remains unclear. Using an unbiased single-cell transcriptomic analysis combined with functional assays, we reveal an unappreciated diversity among thymic iNKT cells, especially among iNKT1 cells. Mathematical modeling and biological methods unravel a developmental map whereby iNKT2 cells constitute a transient branching point toward the generation of iNKT1 and iNKT17 cells, which reconciles the two previously proposed models. In addition, we identify the transcription co-factor Four-and-a-half LIM domains protein 2 (FHL2) as a critical cell-intrinsic regulator of iNKT1 specification. Thus, these data illustrate the changing transcriptional network that guides iNKT cell effector fate.

\section{INTRODUCTION}

Type I or invariant (i) Natural Killer T (iNKT) cells are a versatile population of thymus-derived $\alpha \beta T$ cells that play a critical role in the initiation and orchestration of immune responses in many pathological contexts including infection, cancer, inflammation, and metabolic disorders (Bendelac et al., 2007; Godfrey et al., 2010). iNKT cells respond to lipid-based antigens presented by the quasi-monomorphic major histocompatibility complex (MHC) class I-related CD1d molecule (Bendelac et al., 2007). Their swift response in the periphery is largely dependent on the existence of discrete pre-set subsets that secrete substantial amounts of either interleukin-4 (IL-4), IL17A/F or interferon- $\gamma($ IFN- $\gamma$ ) akin to MHC-restricted T-helper cells and innate lymphoid cells (ILCs). This functional segregation is believed to be instigated in the thymus during their development involving many cues such as self-antigen recognition, transcription factors (TFs), cytokine receptor signaling, and cell-cell interactions.
The developmental steps underlying iNKT cell differentiation remain controversial. Two non-mutually exclusive models have been proposed. Initially, a linear maturation model has been reported (Benlagha et al., 2002; Pellicci et al., 2002) in which newly selected stage $0\left(\mathrm{CD} 24^{+}\right)$iNKT cells mature through stages 1-3 with the loss of CD24 expression and the sequential acquisition of CD44 and NK1.1. Functionally, iNKT cell maturation through these stages is associated with a reduced capacity to produce IL-4 and concomitant increase in IFN- $\gamma$ production, which implies that IL-4-producing iNKT cells (comprised in stage 2) constitute an immature pool of cells. A more recent lineage differentiation model (Lee et al., 2013) suggests that discrete functional iNKT subsets develop from CD24 ${ }^{+}$iNKTO (equivalent to stage 0 ), based on key TF expression into iNKT1 (IFN- $\gamma^{+}, \mathrm{T}-$ bet $^{+}$PLZF ${ }^{\text {lo }}$ ), iNKT2 (IL-4 ${ }^{+}$, PLZF ${ }^{\text {hi }}$ ), or iNKT17 (IL-17 ${ }^{+}, \mathrm{ROR} \gamma \mathrm{t}^{+}$ $\left.\mathrm{PLZF}^{\text {int}}\right)$. In this latter model, the three subsets appear to derive from a common $\mathrm{CCR} 7^{+}$intermediate progenitor (Wang and Hogquist, 2018). Although these findings constitute great strides, we still lack a clear understanding of the developmental steps 
governing iNKT cell development and their effector differentiation, at both the cellular and molecular levels.

Recent advances in genomic profiling have provided insights into the highly divergent gene programs of thymic iNKT subsets (Engel et al., 2016; Georgiev et al., 2016; Lee et al., 2016). However, single-cell approaches have used a limited number of cells and/or relied on iNKT0/1/2/17 subsets pre-sorted based on cellsurface markers. Although invaluable, these approaches precluded a comprehensive analysis of iNKT cell heterogeneity and possibly omitted functionally relevant subsets or intermediate precursor cells.

In an attempt to further our understanding of the dynamics and checkpoints that dictate iNKT cell differentiation and sublineage commitment, we generated the transcriptomic profile of a large number of total thymic iNKT cells using single-cell (sc) RNA sequencing (scRNA-seq) in an unbiased fashion. In addition to the described iNKT0/1/2/17 subsets, unsupervised computational analysis of the transcriptomes uncovered previously unappreciated additional heterogeneity within iNKT cells, including several iNKT1 subsets. Moreover, by combining mathematical algorithms and biological assays, we propose a model for iNKT cell effector differentiation in which iNKT1 and iNKT17 subsets derive from iNKT2. Moreover, iNKT1 subsets arise linearly and sequentially from iNKT2 cells. Finally, we define FHL-2, as an important molecular actor in iNKT1 effector fate. Thus, our data provide a comprehensive understanding of iNKT thymocyte heterogeneity and the transcriptional events that dictate sublineage decisions.

\section{RESULTS}

ScRNA-Seq Indicates Substantial Heterogeneity in iNKT Thymocytes

To reveal the transcriptomic profile of iNKT thymocytes in an unbiased manner, we purified total iNKT cells (live CD3 ${ }^{+}$PBS57/ CD1d tetramer ${ }^{+}$cells) from juvenile (5 week old) C57BL/6J mice (Figure S1) and subjected them to droplet-based scRNAseq. After control/filtering steps (Figure S1; STAR Methods), 3,290 cells were considered for further analyses. Unsupervised computational analysis, using the Louvain algorithm (Kiselev et al., 2017) indicated the existence of nine discrete clusters (I-IX) with different prevalence (Figure 1A). Comparison of the expression pattern of genes in these communities (Figure 1B; Table S1) to published reports (Engel et al., 2016; Georgiev et al., 2016; Lee et al., 2016) indicated that most of these clusters matched with the previously identified iNKT0, iNKT1, iNKT2, and iNKT17 subsets. Differentially expressed genes (DEGs) in cluster I (1.2\% of total iNKT cells) included Lef1, Itm2a, Ccr9, Id3, and Ldhb (Figures 1B and 1C; Table S1), several genes highly related to iNKTO (Engel et al., 2016). Cluster II (2.5\% of total iNKT cells) was enriched for many genes involved in cytoskeletal control (Stmn1, Ska1, Cfl1), mitochondrial activity (Atp5j, Atpif1, Cox5b, Cox6a1), apoptosis regulation (Birc5, Set, Sod1), and mitosis (Cenpe, Ccnb1, Cdk1, Cdc20) (Figure 1B; Table S1) indicative of cell proliferation in either functional $S$ or G2M phases (Figure 1D) and was therefore named "cycling iNKT." Clusters III (7.4\% of total) and IV (12.3\% of total) displayed high similarities characterized by a high expression of genes associated with iNKT2 biology such as Zbtb16, Plac8, I/17rb, and Icos (Figures $1 \mathrm{~B}$ and $1 \mathrm{E}$; Table $\mathrm{S} 1$ ) and were therefore referred to as iNKT2a and iNKT2b, respectively (Engel et al., 2016; Lee et al., 2016). Cluster V (2.7\% of total) was homolog to iNKT17 cells as illustrated by the high expression of $/ 11 r 1$, Rorc, Ccr6, Tmem176a, and Emb (Figures 1B and 1F; Table S1). iNKT1 cells were comprised within clusters VI-VIII $(64.6 \%$ of total) based on their higher expression of Id2, Nkg7, Klrb1c, KIrd1, and II2rb (Engel et al., 2016) (Figures 1B and 1G; Table S1). The last cluster (cluster IX, 9.1\% of total) exhibited a unique and unexpected signature featuring many interferon-stimulated genes (ISGs) such as Ifit3, Isg15, Stat1, and Irf7 (Table S1) and thus was referred to as iNKT-ISG. As expected, the identified clusters showed differential and/or selective expression of some signature TFs such as Rorc, Tbx21, and Zbtb16 (Lee et al., 2013) (Figure 1H). Altogether, this analysis indicates a previously unappreciated transcriptional heterogeneity in iNKT thymocytes.

\section{Gene Signature Associated with iNKT Thymocyte Clusters}

Newly selected iNKTO cells are believed to generate all effector subsets (Benlagha et al., 2002, 2005). Many genes encoding for proteins strongly associated with $\mathrm{T}$ cell receptor (TCR) signaling were enriched in iNKT0 cells (cluster I) such as Pdcd1 (encoding for PD-1), Cd27, Cd28, Slamf6, and Cd81 (Table S1). Several TF-encoding genes reported to control iNKT development were also upregulated in iNKT0 cells such as Sox4 (Malhotra et al., 2018), Lef1 (Carr et al., 2015), Id3 (Li et al., 2013), and Tox (Aliahmad and Kaye, 2008). Consistent with a role for CCR9 in thymocyte development and thymic trafficking (Uehara et al., 2002), Ccr9 transcripts were upregulated in iNKTO cells (Table S1). In addition, a large proportion of DEGs in iNKTO encoded for products involved in nucleic acid regulatory processes and epigenetic landscape (Table S2) suggesting that iNKTO cells may undergo intense genome reprogramming.

In line with a role for miRNA in iNKT development (Henao-Mejia et al., 2013; Koay et al., 2016), Drosha, a key gene in miRNA biogenesis (Lee et al., 2003), was upregulated in both iNKT2 clusters (III and IV). Comparative analysis between iNKT2a and iNKT2b transcriptomes indicated that iNKT2a was enriched for genes related to tissue emigration such as KIf2 and S100a4 (Weinreich and Hogquist, 2008) (Table S3A) that may indicate the presence of potential iNKT thymic emigrants as recently proposed (Wang and Hogquist, 2018). Ccr7 transcripts were also enriched in iNKT2a (Wang and Hogquist, 2018). Surprisingly, several DEGs typically associated with iNKT17 (Engel et al., 2016) were enriched in iNKT2a, including Lmo4, Rora, Emb, and Cxcr6 (Table S3A). This suggests that some cells within the iNKT2a cluster have iNKT17 potential. Similarly, iNKT2b cells (cluster IV) expressed higher levels of iNKT1 markers (Engel et al., 2016) than iNKT2a, such as KIrb1c, II2rb, and Nkg7 (Table S3A).

Among iNKT17 signature genes (Table S1) were transcripts whose products are involved in tissue residency (Itgb7, Ccr6, Ccr2, Aqp3). In line with their anti-infectious role (Paget and Trottein, 2013), transcripts encoding for cytokine receptors were enriched in iNKT17 such as $/ / 17 r e, \| / 1 r 1, / / 18 r 1$, and $/ / 7 r$. Interestingly, the percentage of mitochondrial RNAs (Figure S2A) were 
A

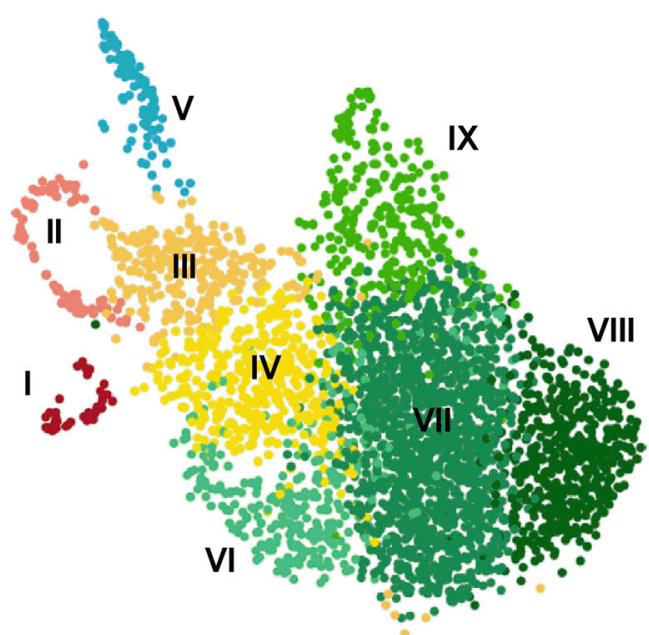

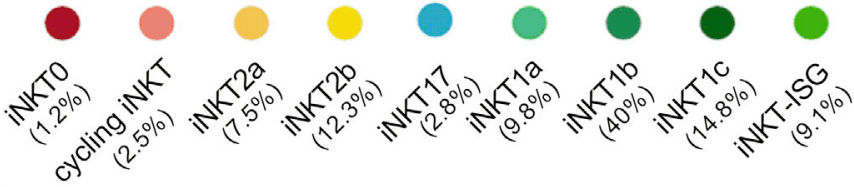

B

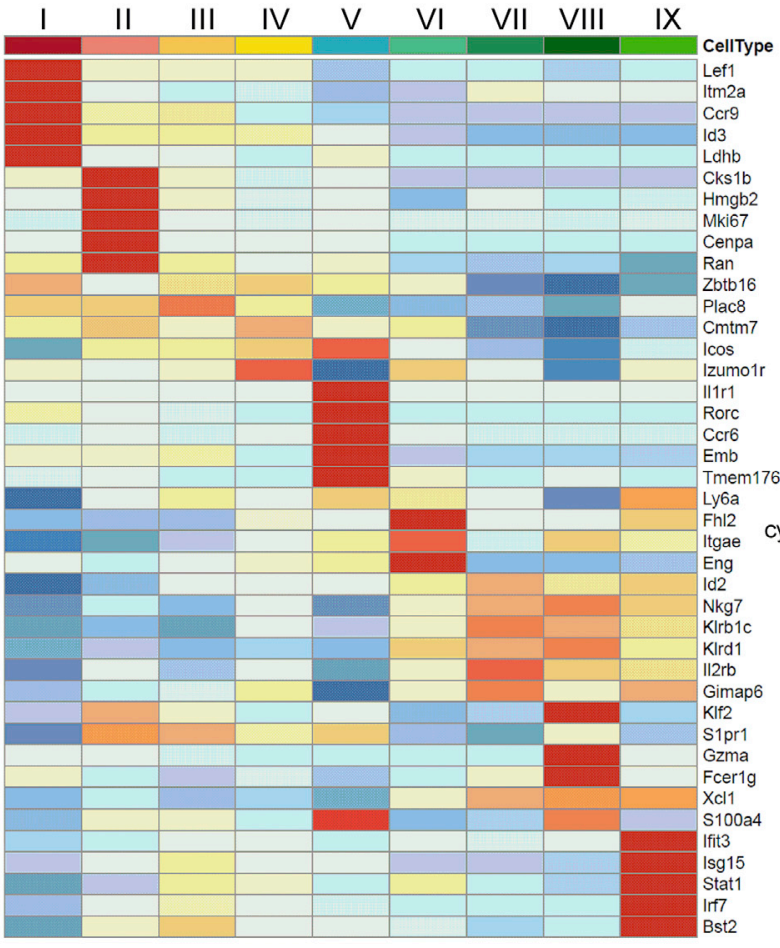

$\therefore \times 0.0 \times 5 \times 2$
C

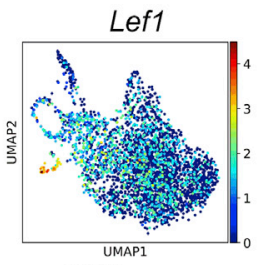

D

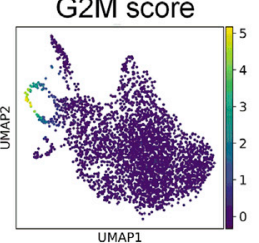

E

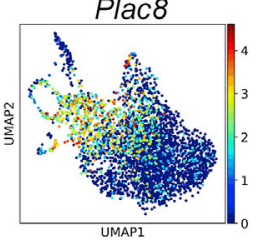

$\mathbf{F}$

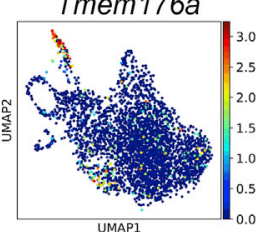

G
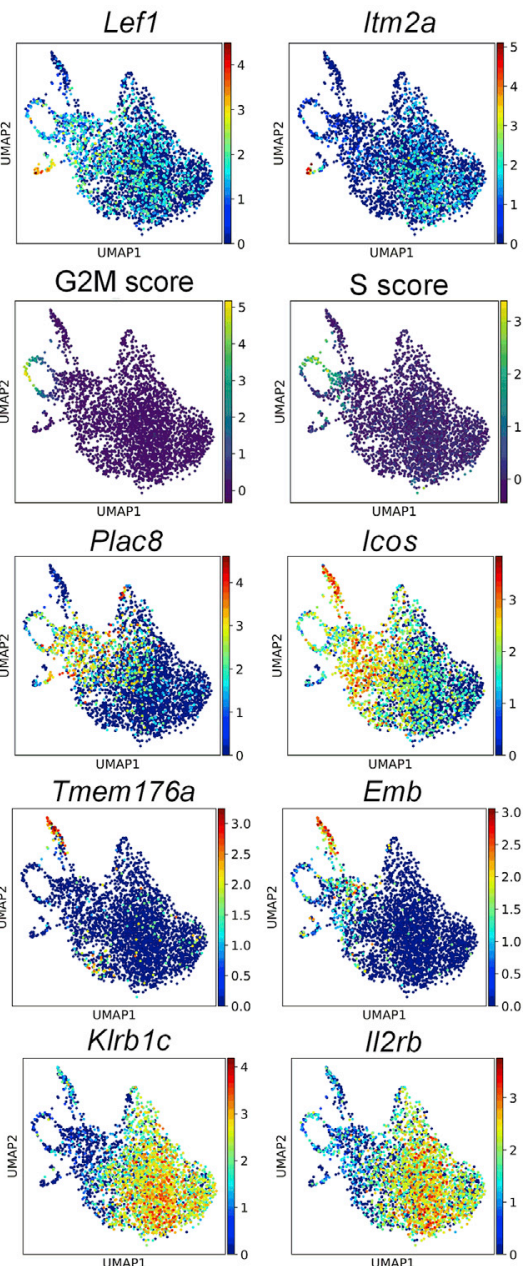

S score

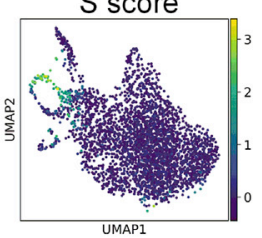

Icos

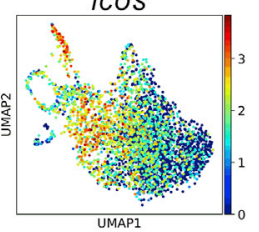

$E m b$

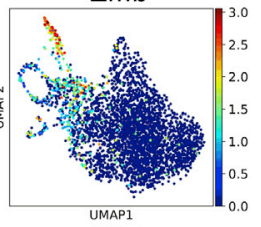

$1 / 2 r b$

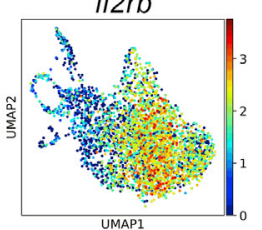

H

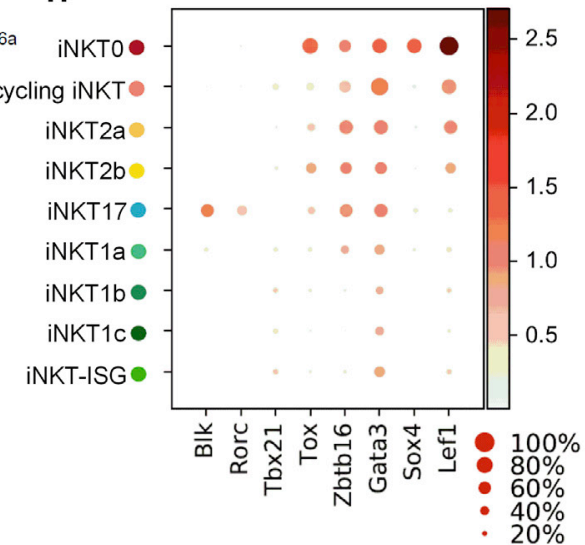

Figure 1. scRNA-seq Identifies Nine Divergent Thymic iNKT Subsets

(A) Identification of cell clusters in scRNA-seq data obtained from C57BL/6J total iNKT thymocytes using the graph-based Louvain algorithm (resolution $=1.5$ ) on Uniform Manifold Approximation and Projections (UMAP). Each dot represents one cell (3,290 cells).

(B) Expression pattern of a representative list of cell subset-specific gene markers.

(C and E-G) Expression of selected gene markers in iNKT0 (C), iNKT2 (E), iNKT17 (F), and iNKT1 (G) identified in (A).

(D) Representation of the quantitative scores for G2M and S phases for each cell.

(H) Dot map showing the expression of 8 selected genes encoding for key transcription factors in iNKT development per cluster. Color gradient and size of dots indicate gene expression intensity and the relative proportion of cells (within the cluster) expressing each gene, respectively. 
enriched in iNKT17 suggesting particular energy requirements for the acquisition of iNKT17 effector functions. Analysis of mitochondrial mass and membrane potential confirmed the enhanced mitochondrial activity in iNKT17 thymocytes (Figure S2B).

As previously mentioned, iNKT1 cells segregated into 3 clusters (VI-VIII) with discrete gene programs (Figure 1B). iNKT1a cells (cluster VI) presented many genes involved in RNA stability (Npm1, Ubb, Cirbp, Pabpc1, Zfp36/2, and Zfp36/1) and translation activity (Eif3e, Eif4a2, Eif3h, Eif1b, Eif1, Eef1b2, Eef1a1, Eef1g, and Eef2) (Table S1). The iNKT1b cluster (cluster VII) comprised most of the iNKT1 cells (54.3\%) and was characterized by the preferential expression of members from the GTPase of the immunity-associated protein (GIMAP) family (Gimap1/3/4/ 5/6) (Table S1), a family of enzymes associated with $T_{H} 1$ differentiation and apoptosis regulation (Engel et al., 2016; Filén and Lahesmaa, 2010). Other genes associated with apoptosis regulation were also enriched in iNKT1b such as Bc/2, Pdcd4, and Cd44. iNKT1b also displayed enriched expression of Cxcr3 and Itgb2 (encoding a subunit of LFA-1) that may indicate a pool of long-term resident cells. iNKT1c cells (cluster VIII) displayed a cytotoxic profile characterized by high expression of Gzma/b, Ccl5 and many transcripts for Killer cell lectin type receptors such as Klra1, KIra5, Klra7, KIra9, Klrc1, KIrc2, Klrd1, Klre1, and KIrk1 (Table S1). Hence, these data confirmed iNKT cell lineage and sublineage-specific transcriptomic profiles and unraveled profiles reminiscent of differential functional activity.

\section{The Clustering of Cycling iNKT and iNKT-ISG Is}

Influenced by Their Biological State

Among the nine clusters, two of them displayed strong enrichment for genes associated with a particular biological state/function such as proliferation (cycling iNKT) and type I IFN response (iNKT-ISG) (Table S1). Thus, we questioned whether their clustering was the consequence of particular biological states rather than a specific stage of differentiation or a functional subset. To address this possibility, subclustering of these two communities were analyzed in a semi-supervised manner using a gene set containing the top 50 DEGs in the other seven iNKT clusters as they may represent more iNKT-specific genes.

This resulted in the emergence of three subclusters $(0,1$ and 2$)$ (Table S3B) within cycling iNKT. Importantly, these subclusters were not associated with a particular phase of the cell cycle (Figure S3A). Rather, subclusters 0 and 1 presented high transcriptomic similarities with iNKTO, iNKT2b, and iNKT2a, while subcluster 2 contained cells more related to iNKT1 clusters (Figure S3B). In addition, monitoring of proliferative iNKT cells by means of mKi67 protein expression indicated that all effector subsets could cycle confirming the heterogeneous nature of cycling iNKT cells (Figure S3C).

Subclustering of iNKT-ISG also generated three subclusters (Table S3C; Figure S3D) that resemble to either iNKT1 (cluster 0), iNKT2/17 (cluster 1), and iNKT2/1a/17 (cluster 2) (Figure S3E). In an attempt to track iNKT-ISG, expression of CD317 (Bst-2), an ISG (Neil et al., 2008) that emerged as a top 15 iNKT-ISG marker gene (Table S1) was assessed on iNKT thymocytes. Consistent with the transcriptomic data, CD317 staining allowed to mark $\sim 10 \%$ of iNKT cells. Using common subset markers, we observed that CD317 ${ }^{+}$iNKT cells displayed a heterogeneous phenotype reminiscent with iNKT1, iNKT2, and iNKT17 cells in roughly similar proportions than their CD317 ${ }^{-}$counterparts (Figure S3F). These observations may suggest that type I IFNsignaling is involved in iNKT cell commitment/differentiation. Interestingly, Ifnar $1^{-1-}$ mice displayed a reduction in iNKT1 and iNKT17 thymocyte numbers, while iNKT0 and iNKT2 remained unaffected (Figure S3G).

Altogether, these results suggest that, once proliferationrelated genes and ISG are removed, cycling iNKT and iNKTISG cells do not present discrete gene signatures and tend to merge with the other defined clusters. Since these two clusters appeared to be driven by their biological activity, they have been removed from the rest of our study.

\section{scRNA-Seq Reveals the Developmental Path of iNKT Thymocytes}

To assess similarities between clusters, we generated a clustermap using the Pearson's method to order clusters by linkages based on similarity between the correlations (Figure 2A). iNKT0 had the most unique signature albeit displaying some similarities with iNKT2a. As expected, iNKT1 and iNKT2 clusters formed two distinct groups (Figure 2A). Of note, iNKT17 appeared closely related to iNKT2 (Figure 2A). Within iNKT1 clusters, iNKT1a and iNKT1b displayed highest similarities (Figure 2A).

To investigate the dynamics and trajectories underlying the iNKT cell-differentiation program, we attempted to create a pseudo-time ordering of iNKT cell transcriptomes to map progenitors to fates. The use of partition-based graph abstraction (PAGA) (Wolf et al., 2019), in an unsupervised manner, enabled to generate a graph-like-map based on connectivity between clusters and single-cell embedding (Figure 2B). Based on the literature, iNKTO cells were defined as the root of the pseudo-time ordering. This could be illustrated by the expression of early markers of iNKT positive selection such as Slamf6 and Pdcd1 (Lu et al., 2019) (Figure 2C). In the predicted model, the PAGA path suggested that iNKTO cells gave rise to iNKT2a cells (Figure 2B). This transition is accompanied by a strong induction of Plac8 and Ccr7 (Figure 2D), in line with their migration from the cortex to the medulla (Wang and Hogquist, 2018). Then, iNKT2a appeared as a node that split into two branches leading to either iNKT17, as illustrated by Rorc expression (Figures 2B and 2E) or iNKT2b, which was associated with the upregulation of Izumo1r (Figures 2B and 2F). Importantly, emergence of several iNKT1 marker genes such as Nkg7 and KIrb1c (Engel et al., 2016) (Figure $2 \mathrm{G}$ ) were found in iNKT2b. Strikingly, iNKT2b appeared to differentiate toward iNKT1 cells with the iNKT1a cluster as the earliest defined iNKT1 subset (Figure 2B). Pseudo-time progression within the iNKT1 branch led to the sequential emergence of iNKT1b and iNKT1c clusters (Figure 2B), illustrated by Tbx21 upregulation and paralleled with a loss of Zbtb16 (Figure $2 \mathrm{H}$ ), an important step for iNKT1 differentiation (Pobezinsky et al., 2015). iNKT1c emerged as a terminal stage of differentiation within the iNKT1 sublineage associated with high expression of Gzmb and KIrc2 (encoding for NKG2C) (Figure 2l). Thus, this model predicts a cascade of molecular events that parallel and potentially drive the differentiation of effector iNKT cells in which iNKT2 clusters appear poised at the crossroad of iNKT sublineages. 
A

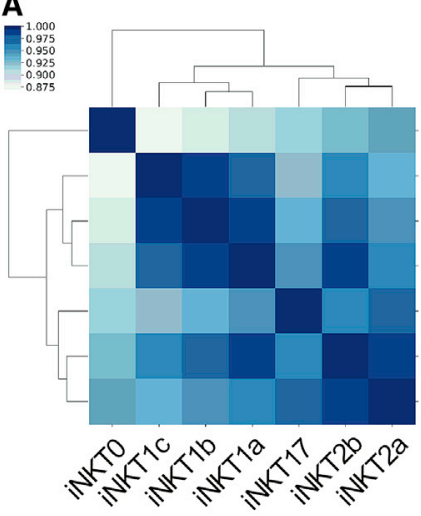

B

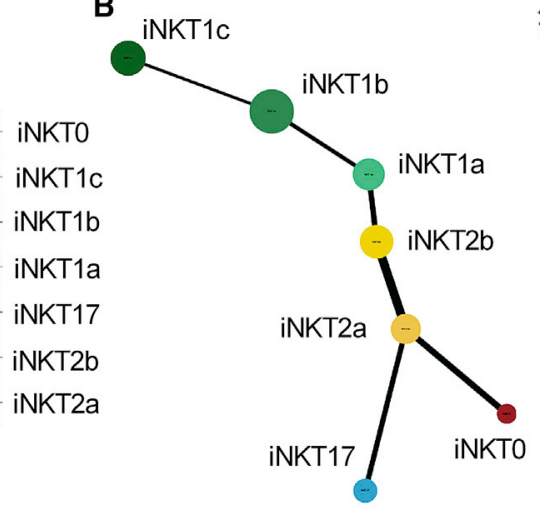

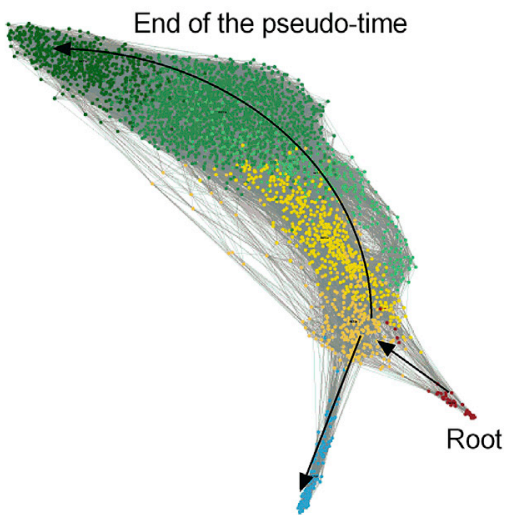

End of the pseudo-time
C

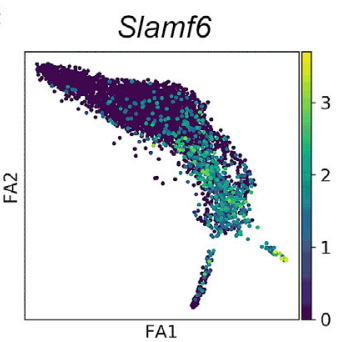

E

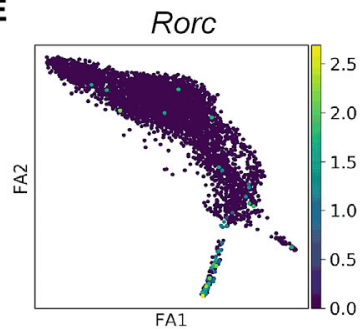

H

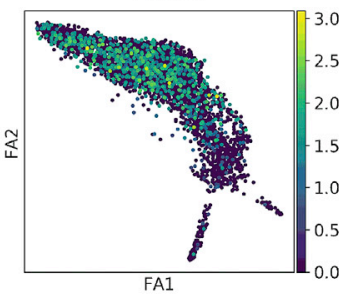

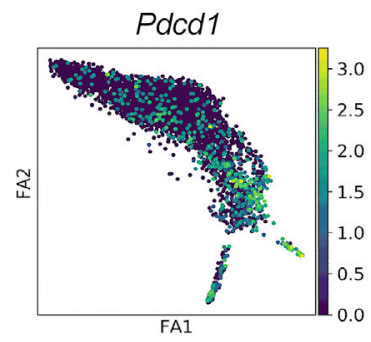

$\mathbf{F}$

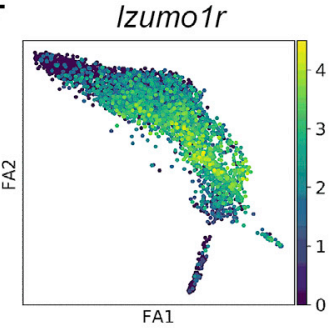

Zbtb16

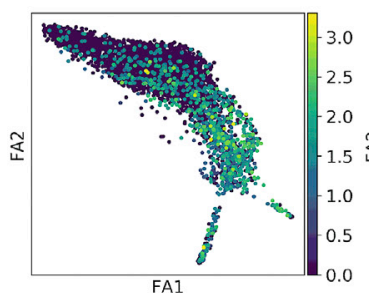

D

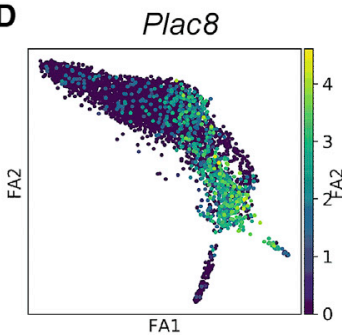

G
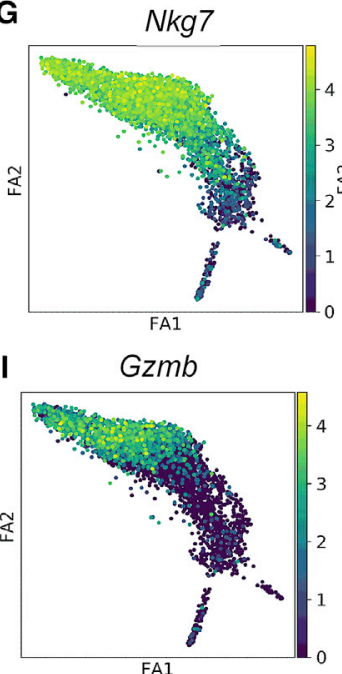

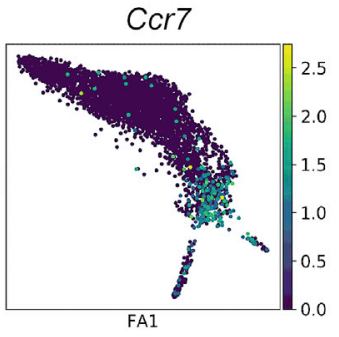

KIrb1c

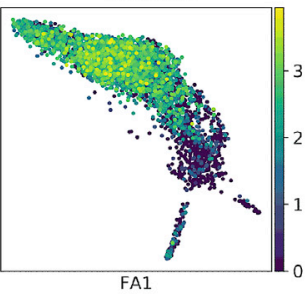

KIrC2

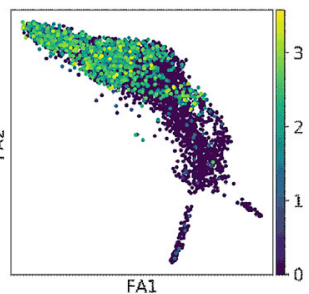

Figure 2. Predictive Developmental Trajectories of iNKT Thymocytes

(A) Clustermap of iNKT subsets comparing each pair of clusters using the Pearson's correlation with hierarchical clustering.

(B) PAGA analysis applied to the scRNA-seq data from $F h / 2^{+/+}$iNKT thymocytes. PAGA graph (left panel) and PAGA-initialized single-cell embedding (right panel) are shown. Weighted edges represent a statistical measurement of connectivity between clusters.

(C-I) Visualization of selected markers on PAGA-initialized single-cell embedding alongside the trajectories defined in (B).

Identification of Thymic iNKT Subsets Based on CellSurface Markers

Building on the scRNA-seq data, we defined a set of surface molecules to track and biologically evaluate these transcriptomically defined thymic iNKT subsets. Based on CD24 (Cd24a), Sca-1 (Ly6a), FR4 (Izumo1r), CD138 (Sdc1), and NK1.1 (KIrb1c) (Figure 3A), we were able to identify iNKT0 (CD24 ${ }^{+}$, Sca-1- ${ }^{-}$, NK1.1'), iNKT2a (CD24- ${ }^{-}$NK1.1- $\left.{ }^{-}, \mathrm{CD}_{138^{-}}, \mathrm{FR} 4^{-}\right)$, iNKT2b

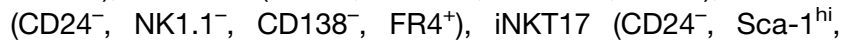

$\mathrm{CD} 3^{+}$), iNKT1a (CD24- $4^{-}$Sca-1 $\left.{ }^{\text {hi }}, \mathrm{CD}_{138^{-}}, \mathrm{NK} 1.1^{\mathrm{dim}}\right)$, iNKT1b $\left(\mathrm{CD} 24^{-}, \mathrm{Sca}-1^{+}, \mathrm{CD}_{138^{-}}, \mathrm{NK} 1.1^{\mathrm{hi}}\right)$ and $\mathrm{iNKT1C}\left(\mathrm{CD} 24^{-}, \mathrm{Sca}^{-1^{-}}\right.$, CD138-, NK1.1 hi (Figure 3B). We compared our gating strategy to the classic maturation stages based on expression of CD44 and NK1.1. As expected, iNKTO were $\mathrm{CD} 24^{+}, \mathrm{NK} 1.1^{-}$, and CD44-/low (stage 0/1) (Figure 3C). Stage $2\left(\mathrm{CD} 24^{-} \mathrm{CD} 44^{+} \mathrm{NK} 1.1^{-}\right)$ cells comprised both iNKT2 subsets as well as iNKT17 cells, some of which can express low levels of NK1.1 (Figure 3C). Notably, iNKT2b displayed a slightly brighter expression of 
A

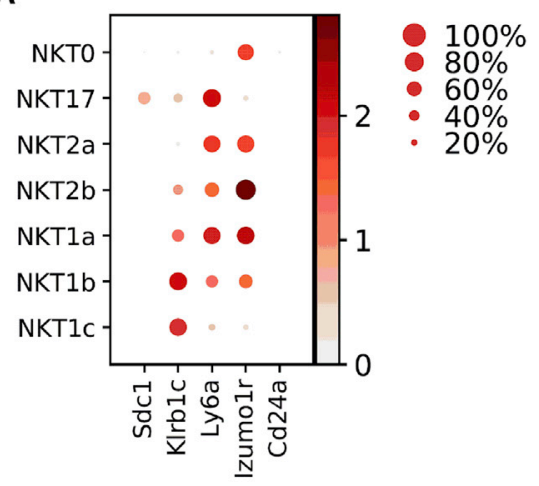

B

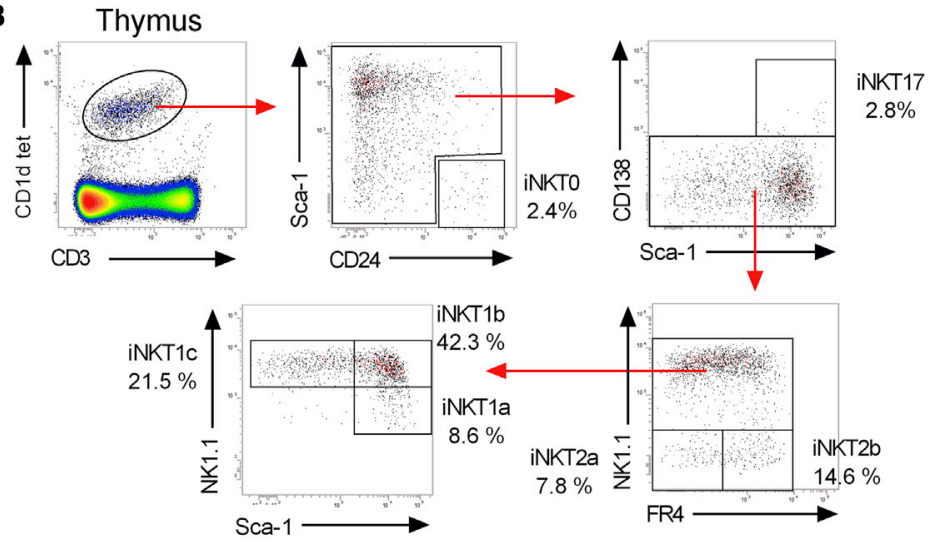

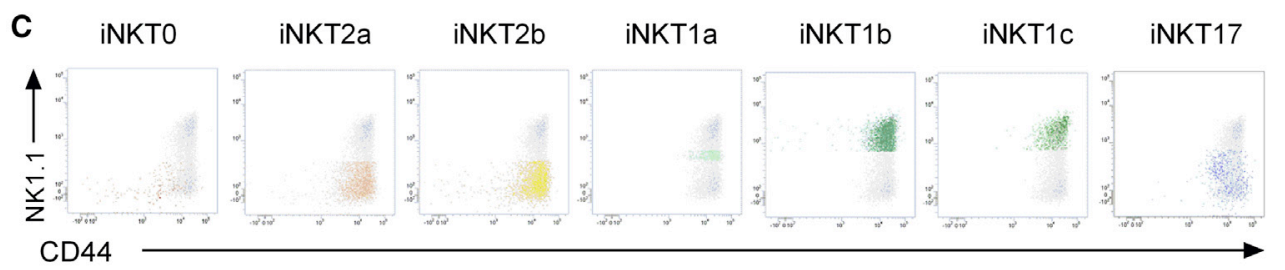
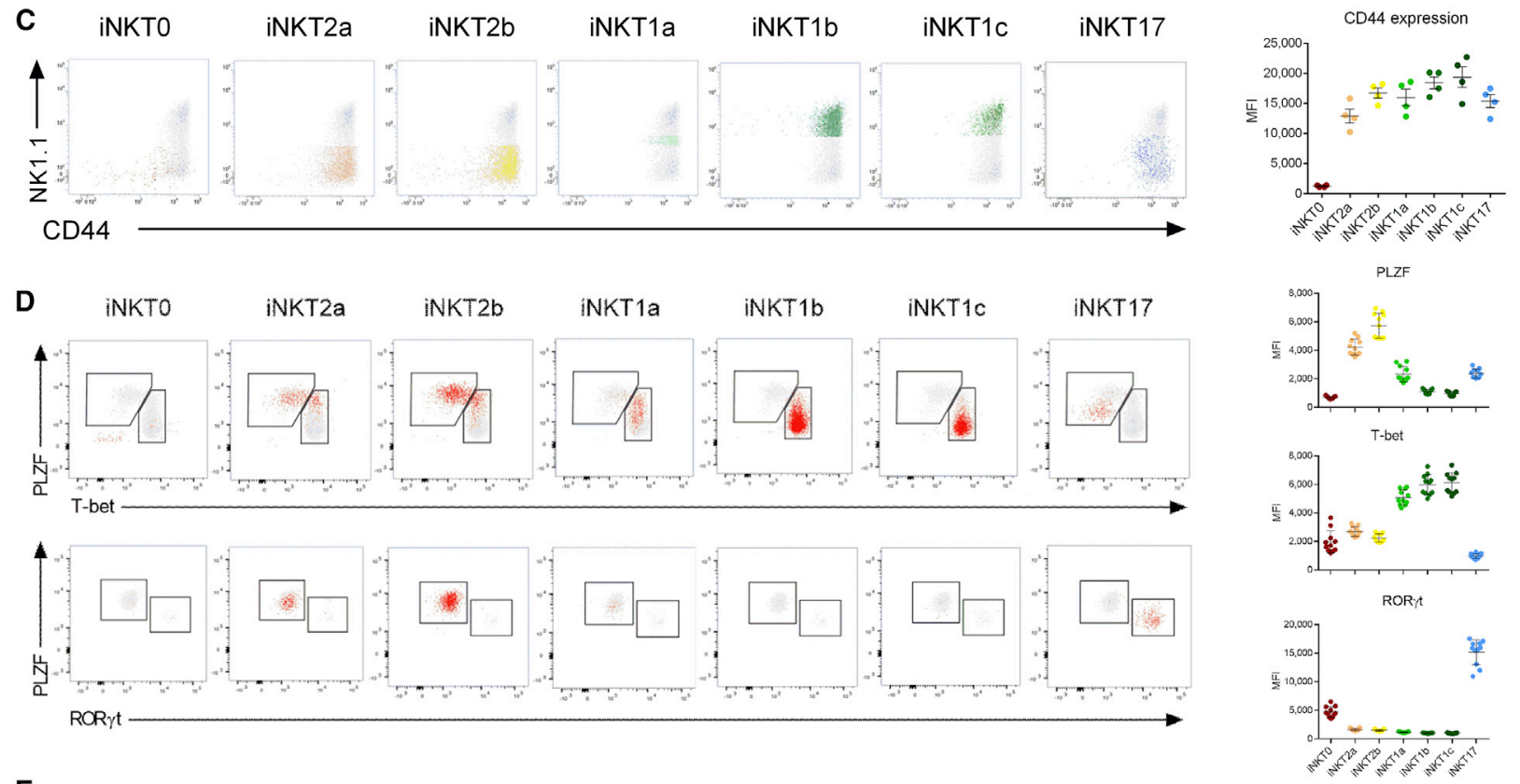

E
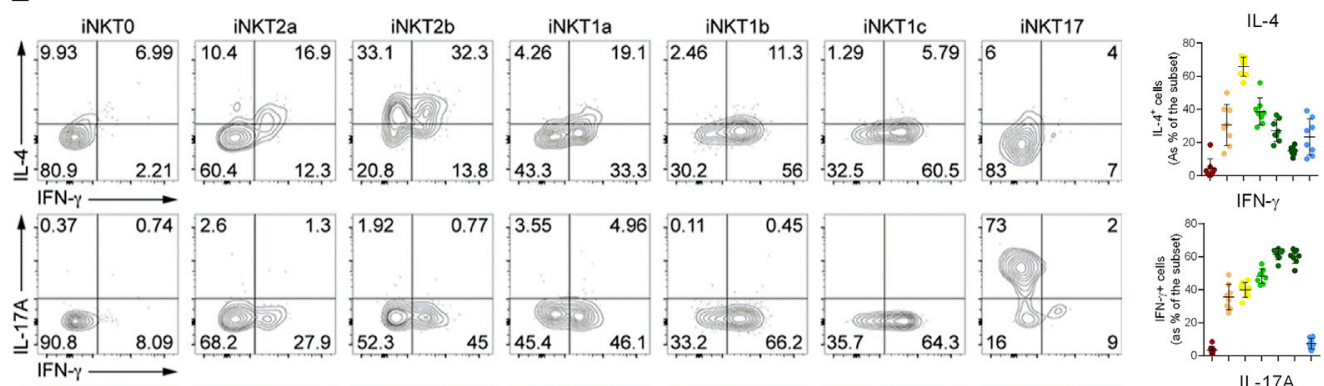

$\mathbf{F}$

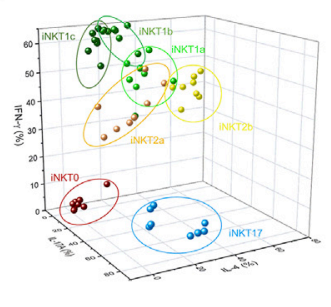

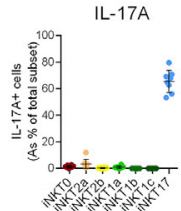

Figure 3. Identification of iNKT Clusters Using Cell-Surface Markers

(A) Dot map showing the expression of 5 surface markers per cluster versus all others. Color gradient and size of dots indicate gene expression intensity and the relative proportion of cells (within the cluster) expressing each gene, respectively. 
CD44 compared to iNKT2a (Figure 3C). All subsets of iNKT1 displayed a stage 3 phenotype $\left(\mathrm{CD} 44^{+} \mathrm{NK} 1.1^{\mathrm{dim} /{ }^{+}}\right.$) (Figure $\left.3 \mathrm{C}\right)$.

The differential expression of PLZF, T-bet, and ROR $\gamma$ t (Lee et al., 2013) also confirmed our assignment. iNKT0 cells were $\mathrm{PLZF}^{-}, \mathrm{T}_{\text {-bet }}{ }^{-}$, and ROR $\gamma \mathrm{t}^{\text {dim }}$ (Figure 3D). As expected and as compared to DP thymocytes, iNKTO cells expressed higher levels of CD69 (Figure S4A). Both iNKT2 subsets expressed high levels of PLZF although its expression was slightly elevated in iNKT2b (Figure 3D). Analysis of classic iNKT2 surface markers (Terashima et al., 2008; Wang and Hogquist, 2018) indicated that both subsets expressed IL-17RB and PD-1 (Figure S4B). iNKT2 subsets expressed low levels of T-bet and no ROR $\gamma$ t (Figure 3D). iNKT17 cells expressed low levels of T-bet, intermediate levels of PLZF, and the highest levels of ROR rt (Figure 3D). All iNKT1 subsets expressed T-bet albeit at various intensities (Figure 3D). In line with our proposed model of differentiation, a gradual decrease in PLZF expression was paralleled with T-bet acquisition in iNKT1 subsets (Figure 3D).

We also tested the cytokine profile of these subsets of iNKT thymocytes upon short-term in vitro stimulation with PMA/ionomycin. As expected, both iNKT2 subsets produced IL-4, although iNKT2b produced higher levels as compared to iNKT2a (Figure 3E). iNKT17 almost exclusively produced IL-17A and iNKT1 subsets produced IFN- $\gamma$ (Figure 3E). However, in line with a previous report (Cameron and Godfrey, 2018), we observed that bona fide iNKT1 - especially iNKT1a-and iNKT17 cells could produce IL-4 at some extent (Figure 3E). Consistent with the proposed developmental trajectories, a decrease in IL-4 production capacity among iNKT1 cells paralleled their maturation/differentiation (iNKT1a > iNKT1b > iNKT1c) (Figure 3E). In line with previous reports (Benlagha et al., 2002; Pellicci et al., 2002), both iNKT2a and iNKT2b subsets co-produced IFN- $\gamma$ and IL-4 but no detectable IL-17A (Figure 3E). Moreover, three-dimensional plotting indicated that iNKT subsets clustered based on cytokine profile (Figure 3F). This analysis suggested that the iNKT2b and iNKT1a are relatively close in terms of cytokine profile (Figure $3 F$ ), and a progression toward a Th1-like profile is observed in iNKT1 subsets (Figure 3G). Thus, the 7 clusters defined by scRNA-seq can be readily identified by flow cytometry using cell-surface markers and have discrete cytokine signature.

\section{Multiple iNKT Subsets Have the Potential to Egress the Thymus \\ The nature of thymus-emigrating iNKT cells remains controversial, and as the field progresses multiple subsets have been reported to leave the thymus including precursors, iNKT2, iNKT17, and iNKT1 cells (Milpied et al., 2011; Pellicci et al., 2002; Wang and Hogquist, 2018). Based on our transcriptomic data, we compared}

the preferential expression of egress/retention gene markers between clusters (Figure 4A). iNKT2a, iNKT17, and iNKT1c clusters were enriched for Klf2, a master transcriptional regulator of sphingosine 1-phosphate receptor (Weinreich and Hogquist, 2008) (Figure 4A; Table S1) that may indicate at least several functional subsets of iNKT thymic emigrants. In addition, several genes of the KLF2 regulon such as Emp3, S100a4, and S100a6 displayed a similar pattern of expression (Figure 4A). Of note, the expression of Ccl5, a regulator of Klf2 expression (Kroetz and Deepe, 2011), was also found elevated in iNKT1c (Table S1). Conversely, iNKT0 expressed CD69, while iNKT2b and iNKT1a/b subsets expressed Cxcr3 (Figure 4A), two genes encoding for proteins involved in thymic retention (Drennan et al., 2009; Weinreich and Hogquist, 2008). To test these observations in vivo, we attempted to block iNKT thymocyte egress by means of FTY720 (Kimura et al., 2018), a selective sphingosine 1-phosphate receptor agonist that induces its internalization (Mandala et al., 2002). Prolonged FTY720 treatment led to a significant increase in iNKT thymocytes (Figure 4B). This artificial retention led to a specific enrichment for iNKT2, iNKT17, and iNKT1c subsets (Figure 4C). Thus, these data may suggest that these three sublineages of functional iNKT cells have the potential to leave the thymus.

\section{iNKT2 Subsets Can Generate Both iNKT1 and iNKT17 Cells}

To biologically evaluate our predictive developmental model, various purified iNKT subsets (Figure S5) were cultured with Traj $18^{-/-}$total thymocytes in presence of IL-7 and IL-15, two critical factors for iNKT cell survival and differentiation (Matsuda et al., 2002). After 4 days of culture, iNKT2a cells generated iNKT2b, iNKT17, and iNKT1 (Figure 5A) but no iNKTO cells (data not shown). Of note, only $12 \%$ of iNKT2a cells retained their original profile suggesting these cells may be in a transient stage of differentiation (Figure 5A). In line with the predicted computational model (Figure 2A), iNKT2b generated iNKT1 but few iNKT17 as compared to iNKT2a (Figure $5 \mathrm{~A}$ ) suggesting that this very subset mainly lost its plasticity at least in this ex vivo setting. As expected, iNKT1 $a$ and iNKT1b generated large amounts of iNKT1 cells (Figure 5A). However, iNKT1a could still generated a substantial amount of iNKT2 (Figure 5A). Importantly, this effect was much more limited with iNKT1b cells (Figure 5A). Consistent with their proliferative and pluripotent nature (Figure 2B; Figure S3C), cultures with iNKT2a resulted in the generation of higher number of iNKT cells compared to other subsets (Figure 5B).

To monitor time to positive selection of iNKT cells, we took advantage of Rag2 ${ }^{\text {GFP }}$ mice by following the GFP decay as an indicative marker of time from positive selection (Boursalian et al., 2004). iNKTO cells expressed the highest level of GFP

(B) Gating strategy for the identification of the iNKT subsets in the thymus of 5-week-old C57BL/6J mice. Percentages are indicated as a proportion of total iNKT cells.

(C) Representative overlay dot plots showing CD44 and NK1.1 expression in iNKT subsets as obtained in (B) is shown in the left panel. Individuals/means \pm SEM of mean fluorescence intensity (MFI) of CD44 for each subsets are depicted in the right panel. Data are representative of one experiment out of three. (D) Flow cytometry showing PLZF, T-bet (top panel), and ROR $\gamma$ t within PLZF ${ }^{+}$T-bet $^{-}$cells (middle panel) expression in iNKT subsets as identified in (B). Data are representative of three experiments. Individuals/means \pm SEM of mean fluorescence intensity (MFI) for each subsets are depicted in the right panel. (E) IFN- $\gamma$, IL-17A, and IL-4 production in PMA/ionomycin-stimulated iNKT subsets as identified in (B). Of note, iNKT17 were defined as ROR $\gamma \mathrm{t}^{+}$due to downmodulation of Sdc-1 upon stimulation. Data are pooled from two experiments. Individuals/means \pm SEM for each subset are depicted in the right panel. (F) 3D scatterplot for cytokine production (IL-4, IFN- $\gamma$, and IL-17A). Each data point represents one mouse $(n=8)$. Data are pooled from two experiments. 
A

\section{Genes associated to thymic egress or retention}

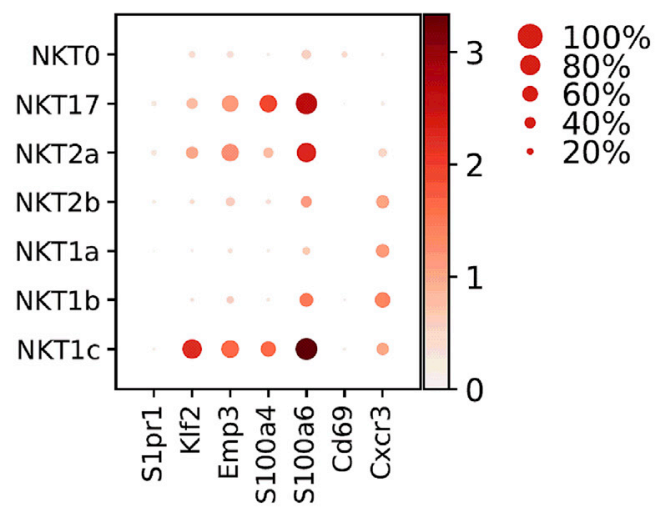

B

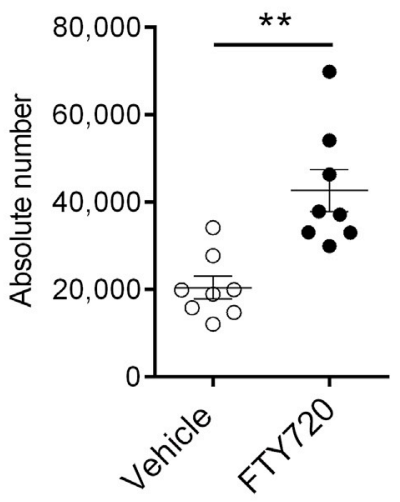

Figure 4. Transcriptomic and Biological Evaluation of iNKT Thymocyte Egress

(A) Dot map showing selected gene expression per cluster versus all other cells. Color gradient and size of dots indicate gene expression intensity and the relative proportion of cells (within the cluster) expressing each gene, respectively.

( $B$ and $C$ ) Mice received daily vehicle (open circles) or FTY720 (filled circles) by the i.p. route during 7 days. Mice were sacrificed on day 8 and thymi were harvested. The absolute number of iNKT cells (B) and subsets (C) were determined by flow cytometry. Individuals/means \pm SEM of pooled data from two experiments are shown ( $n=5$ mice/ group). ${ }^{*} p<0.05 ;{ }^{*} p<0.01$.

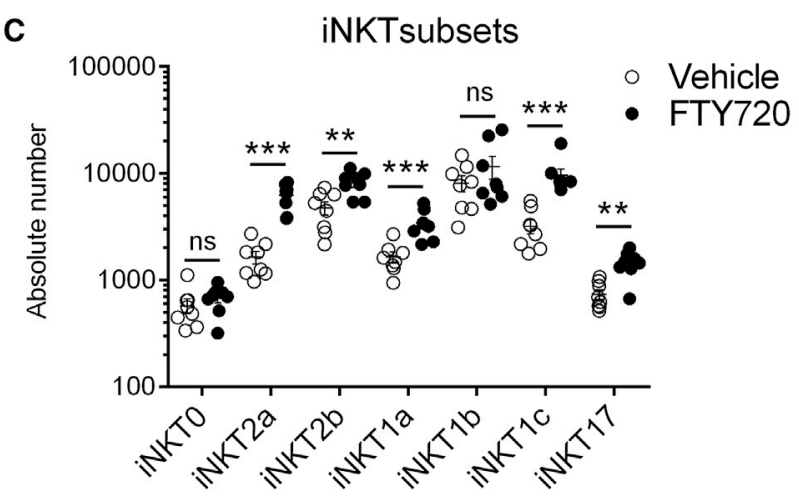

gene encoding for the transcription co-factor Four and a half LIM domain 2 (FHL2) recently shown to control NK cell development (Baranek et al., 2017) but whose function in $\mathrm{T}$ cell biology remains elusive. We found that $F h / 2^{-/}$mice had a decrease in total thymic iNKT cell numbers (Figure 6a). Although a slight decrease in total thymocyte number was noted (Figure S6A), no clear modifications were observed neither on DN subsets nor on CD4SP, CD8SP and DP (Figure S6B). Based on TF expression, we observed that $F h / 2^{-/-}$iNKT thymocytes displayed a reduced number of iNKT1 compared to their $\mathrm{Fhl}^{+/+}$counterparts, while both

indicating recent positive selection (Figure $5 \mathrm{C}$ ). In line with the maturation process, levels of GFP varied between subsets with intermediate levels in iNKT2 and iNKT17 cells but low levels in iNKT1 subsets (Figure 5C). A gradual reduction was observed between iNKT1 subsets reinforcing the possibility that iNKT1a cells were an immature stage in iNKT1 differentiation (Figure 5C).

Since at least some iNKT2 thymocytes appeared as intermediate of development in iNKT effector fate, we assessed how iNKT subset prevalence evolve with age. While iNKT2 cells were the major subsets in 3-week-old thymi, we observed a striking decrease in their frequency as mice aged (Cruz Tleugabulova et al., 2019; Lee et al., 2013) (Figure 5D). Consistent with the thymic resident nature of iNKT1b cells, we observed a strong enrichment for this subset with age (Figure 5D). No major modulation was observed for iNKT0, iNKT1a, and iNKT1c (Figure 5D). Of note, relative proportion of iNKT17 cells was severely decreased in 20-week-old mice (Figure 5D). Altogether, these biological approaches confirm the predictive RNA-based model and indicate that most iNKT2 are pivotal in iNKT commitment toward iNKT1 and iNKT17 profiles.

\section{Fh/2 Controls Commitment to iNKT1 Lineage}

iNKT1a cells appeared as the earliest committed iNKT1 cells (Figure $2 \mathrm{~A}$ ) and therefore it is possible that some of the signature genes within this cluster may be involved in effector fate specification. Among those candidates is Fh/2 (Figure 1B; Table S1), a
iNKT2 and iNKT17 remained unaffected (Figure 6B). Selective iNKT1 cell defects were also observed in peripheral tissues of FHL2-deficient mice (Figure S6C). Using our staining strategy, we observed that the lack of iNKT thymocytes in Fh/2 ${ }^{-1-}$ mice was restricted to iNKT1b and iNKT1c (Figure 6C). Conversely, the number of iNKT2b cells was slightly increased in Fh/2 ${ }^{-1}$ mice (Figure 6C) suggesting a block at the iNKT2b to iNKT1a transition phase.

To evaluate this at the transcriptomic scale, we generated scRNA-seq data from $F h / 2^{-/-}$iNKT thymocytes. By applying graph-based clustering of $F h / 2^{-/-}$iNKT after aggregation of WT transcriptomes, we confirmed the presence of most of the 9 original clusters found in WT mice, with the exception of iNKT1a, which were absent from $F h / 2^{-1-}$ iNKT cells, suggesting the critical role of this gene in the transcriptomic regulation of this cluster (Figures $6 \mathrm{D}$ and $6 \mathrm{E})$. Consistent with the biological data, a reduced proportion within iNKT1 clusters was observed in Fh/2 ${ }^{-/-}$iNKT transcriptomes (Figure 6E). Paralleling their higher relative proportion, several key iNKT2 marker genes were enriched in Fh/2 ${ }^{-1-}$ iNKT cells including Zbtb16, Izomu1r, and Plac8 (Figure 6F; Table S4A). However, cluster-by-cluster analysis did not show any significant modulation for Zbtb16 (Tables S4B-S4F). Post-transcriptional regulation of PLZF is required to allow iNKT1 specification (Pobezinsky et al., 2015). In line, the levels of PLZF protein were significantly increased in $F h / 2^{-/-}$iNKT subsets at the exception of iNKT2a and iNKT17 (Figure 6G). Compared to controls 
A

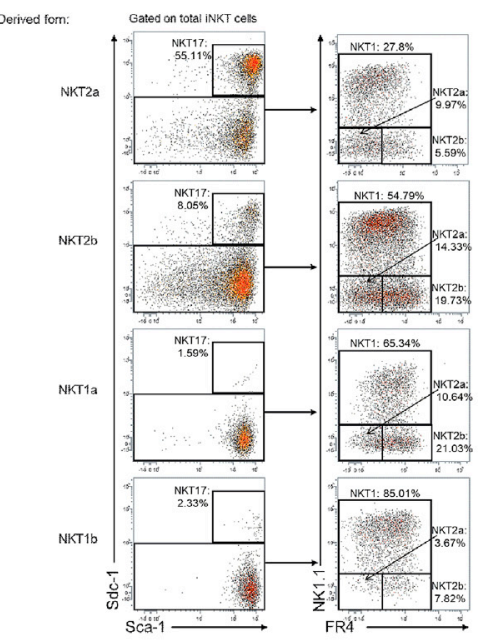

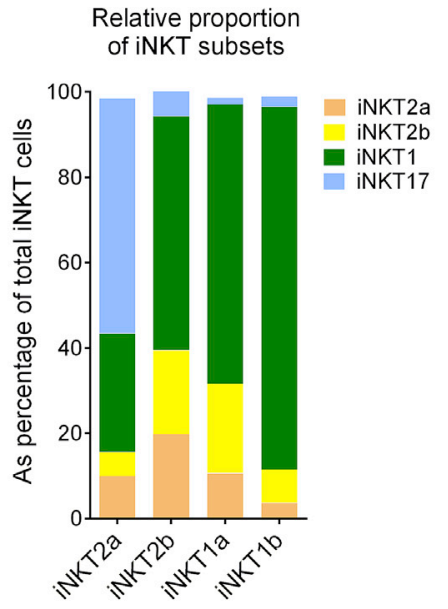

B

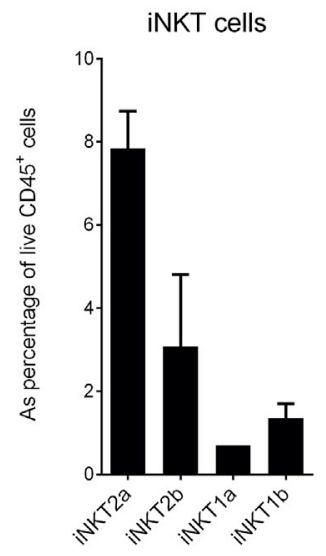

C

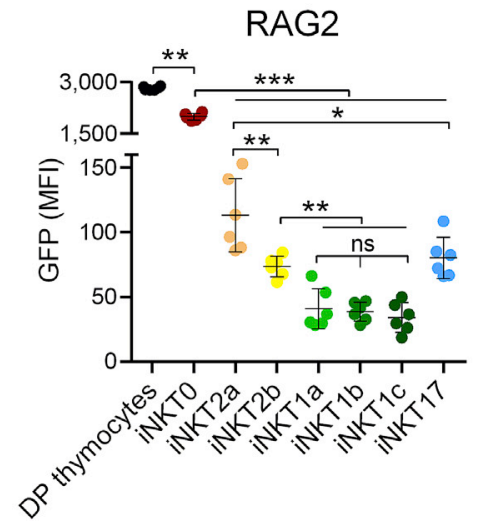

D

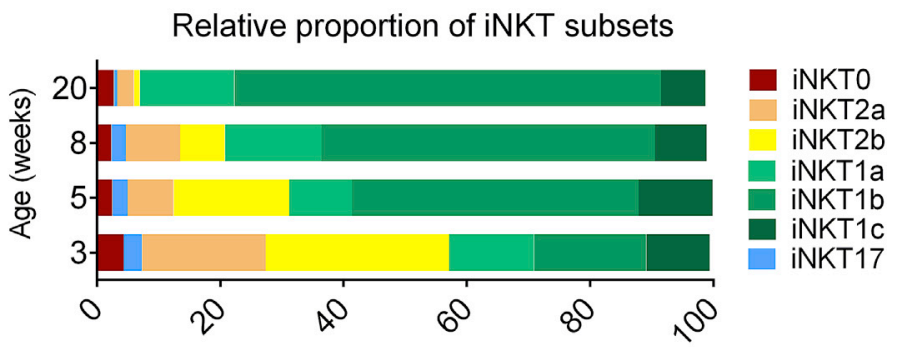

As percentage of total iNKT cells

Figure 5. iNKT1 and iNKT17 Effector Cells Derive from iNKT2 Precursors

(A and B) Purified iNKT subsets were cultured for 4 days with total thymocytes from Traj18 ${ }^{-1-}$ mice in presence of IL-7 and IL-15. (A) Representative dot plots of generated iNKT cells from indicated subsets are depicted in the left panel. Mean of iNKT subsets' relative proportion obtained from three experiments is shown in the right panel. (B) Percentage of total iNKT cells generated within live cells after 4 days of culture was determined by flow cytometry. Mean \pm SEM of three experiments is shown.

(C) Flow cytometry of GFP in iNKT subsets using Rag $2^{\mathrm{GFP}}$ mice. Representative data of two experiments are shown in the top panel. Individuals/means \pm SEM of MFI of GFP are shown. ns, not significant; ${ }^{*} p<0.05 ;{ }^{* \star} p<0.01,{ }^{* \star *} p<0.001$.

(D) Relative proportion of iNKT subsets according to the indicated age from littermate mice. Mean pooled from two experiments is shown $(n=8)$.

(Figure 2B), application of the PAGA algorithm on Fh/2 ${ }^{-/-}$iNKT transcriptomes revealed some disruptions in the interconnectivity between clusters including the iNKT2/iNKT1 transition (Figure $6 \mathrm{H}$ ). Collectively, FHL2 controls iNKT1 lineage commitment possibly through its post-transcriptional regulation of PLZF.

\section{DISCUSSION}

Using high dimensional scRNA-seq and biological assays, we herein characterize the cascade of events occurring in the aftermath of iNKT thymocyte positive selection. This analysis enabled to highlight an unexpected heterogeneity, many of those subsets being transitional stages linked to the acquisition of their effector program. Importantly, we were able to analyze these transient stages through pseudo-time ordering and ex vivo differentiation assays.
This reveals a continuous and dynamic process experienced by differentiating iNKT thymocytes following positive selection. In this process, iNKTO matured to uncommitted iNKT2a cells characterized by expression of Plac8 and Ccr7. At the protein level, this transition phase is accompanied by a strong expression of PLZF. Interestingly, the transcriptional program of iNKT2a is reminiscent to a cluster of central and transitional MucosalAssociated Invariant T (MAIT) thymocytes (Koay et al., 2019; Legoux et al., 2019). Thus, iNKT2a likely represent a precursor stage preceding effector fate commitment as previously suggested (Wang and Hogquist, 2018). In agreement with this, iNKT2a emerged as a pivotal subset that can either egress from the thymus or fulfill their differentiation program. As such, iNKT2a can commit to the iNKT17 or iNKT1 sublineages. Interestingly, commitment to the iNKT1 effector fate seems to require transitional states that closely resemble to iNKT2 (e.g., iNKT2b 
A

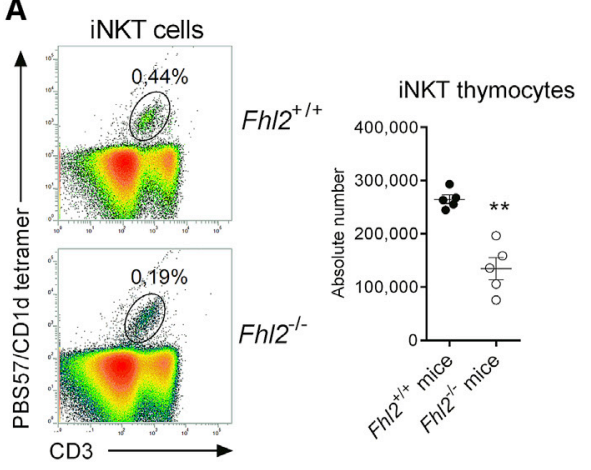

B

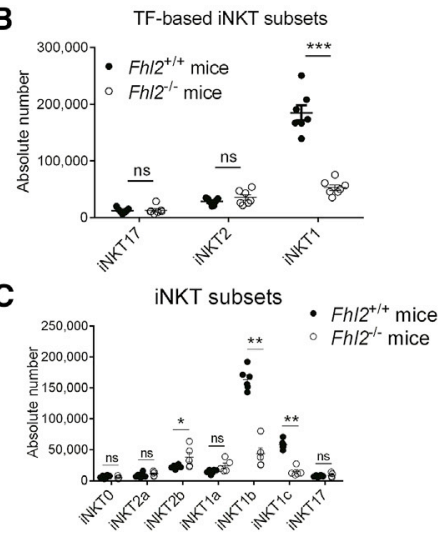

D
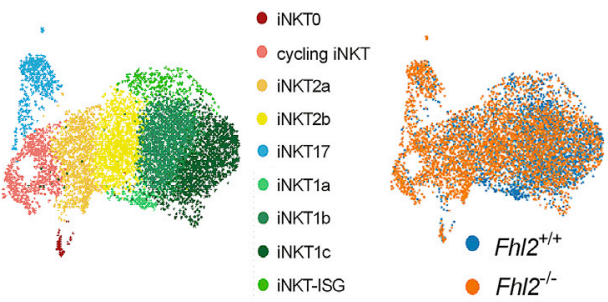

E

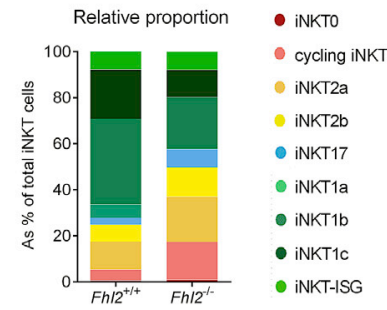

$\mathbf{F}$

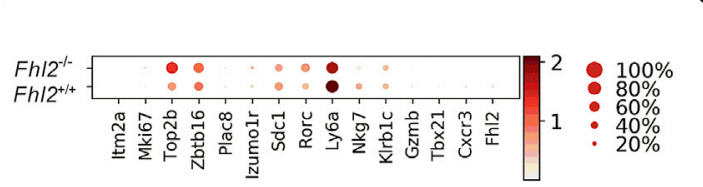

G
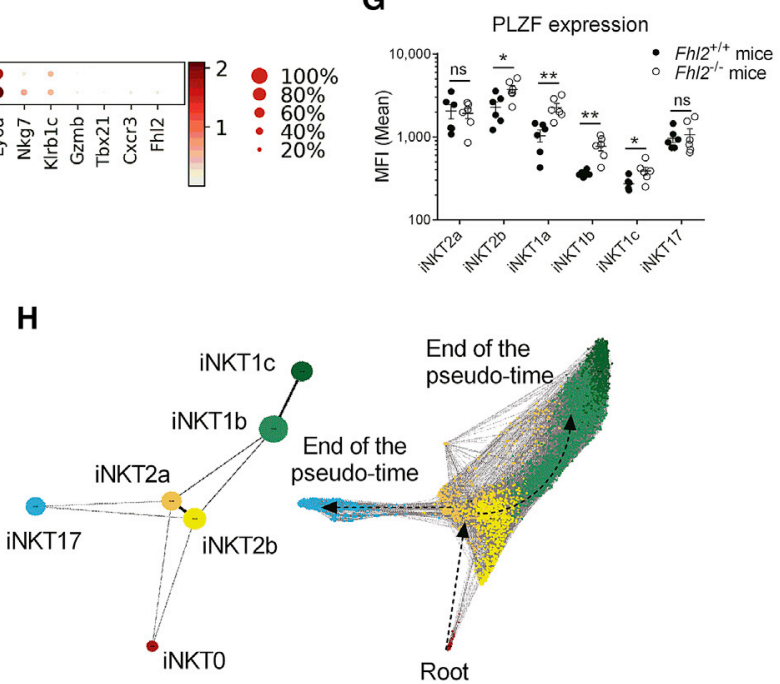

Figure 6. Fh/2 Deficiency Affects iNKT Cell Development

(A) Flow cytometric analyses of iNKT thymocytes in $\mathrm{Fhl}^{+/+}$and $\mathrm{Fh} \mathrm{2}^{-/-}$littermate mice. Representative dot plots from eight experiments are shown in the left panel. Individuals/means \pm SEM of absolute number of iNKT thymocytes from one representative experiment out of eight are shown in the right panel. ${ }^{\star *} p<0.01$.

(B) Flow cytometry of iNKT subsets from $\mathrm{Fh} / 2^{+/+}$or Fhl $2^{-1-}$ mice based on PLZF and T-bet expressions: iNKT17 (PLZF ${ }^{\text {dim/- }}$ T-bet'), iNKT2 (PLZF ${ }^{\text {hi }}$ T-bet ${ }^{-}$), and iNKT1 (PLZF ${ }^{\text {dim/- }}$ T-bet $\left.^{+}\right)$. Data are representative of two experiments. Individual values and means \pm SEM for each subsets are depicted.

(C) Flow cytometry analysis of iNKT subsets in thymi of $\mathrm{Fhl}^{+/+}$and $\mathrm{Fhl}^{-/-}$mice. Individuals/ means \pm SEM for each subsets from two experiments are shown. ns, not significant; ${ }^{* \star \star} p<0.001$. (D) Aggregated UMAP representation of scRNAseq data from $\mathrm{Fhl}^{+/+}$(3,290 cells) and $\mathrm{Fh} / 2^{-/-}$ $(4,189$ cells) iNKT thymocytes. Clusters identities are shown in the left panel, and WT onto KO projection is shown in the right panel.

(E) Relative proportion of iNKT clusters in $\mathrm{Fh}^{2} \mathrm{2}^{-/-}$ and $F h / 2^{+/+}$mice from scRNA-seq data.

(F) DEG in $\mathrm{Fhl}^{+/+}$versus $\mathrm{Fhl} / 2^{-/-}$iNKT thymocytes from scRNA-seq analysis. Dot map depicting selected gene expression per cluster is shown in the bottom panel. Color gradient and size of dots indicate gene expression intensity and the relative proportion of cells (within the cluster) expressing each gene, respectively.

(G) Flow cytometry of PLZF expression in $\mathrm{Fhl}^{+/+}$ and $F h / 2^{-l-}$ iNKT subsets. Individuals/means \pm SEM of PLZF MFI for each subsets from one experiment out of three are depicted. ns, not significant; ${ }^{*} p<0.05 ;{ }^{* *} p<0.01$.

(H) PAGA analysis applied to the scRNA-seq data from $\mathrm{Fhl}^{-1-}$ iNKT thymocytes. PAGA graph (left panel) and PAGA-initialized single-cell embedding (right panel) are shown. Weighted edges represent a statistical measurement of connectivity between clusters.

cytes do not appear to differentiate into bona fide MAIT2 cells (Legoux et al., 2019). In any case, iNKT2 appear to

and iNKT1a). This raises the question whether iNKT2 have to be considered as an intermediate, a terminal stage of differentiation or a combination of both. Previous reports indicated that iNKT2 did not have the potential to give rise to iNKT1 cells (Lee et al., 2013; Watarai et al., 2012). This discrepancy may lie in the phenotypical characterization of iNKT2 cells between the studies. In our ex vivo differentiation model, consistent with pioneer studies (Benlagha et al., 2002; Pellicci et al., 2002) neither iNKT2a nor iNKT2b cells maintained their original stage, although we cannot exclude that a small proportion of these latter can retain a long-term iNKT2 profile. Moreover, we constantly observed that most of the iNKT2 thymocytes co-produced IL-4 and IFN- $\gamma$. It is noteworthy that murine MAIT thymo- conserve a certain degree of plasticity that enables them to generate either iNKT1 or iNKT17 cells, a situation that is less obvious for these two latter subsets. In addition, iNKT2 numbers are strongly reduced with age. Although this favors the hypothesis of a developmental precursor, it can also result from changes in the thymic environment (e.g., cytokine and ligand availability) that may interfere in the iNKT2 differentiation process. Further works will be required to delineate the true nature of iNKT2 in iNKT cell effector fate.

Our data also pointed toward an unappreciated transcriptomic heterogeneity within the pool of iNKT1 cells. First, iNKT1a emerges as a pivotal subset in iNKT1 specification in which the transcription co-factor FHL2 is involved. Its recognized 
repressive activity on PLZF (Tran et al., 2016) may, at least in part, explain the role of FHL2 in iNKT1 effector fate (Pobezinsky et al., 2015). Similarly, the mechanisms that regulate FHL2 expression and activity in iNKT cells deserve further consideration. Moreover, it will be interesting to test the cell-intrinsic involvement of FHL2 in other PLZF-dependent immune cells such as ILC, MAIT, or $\gamma \delta T$ subsets. Importantly, FHL2 has been shown to interact with several key factors in lymphocyte development such as Id2, Id3, and Nur77 (Tran et al., 2016). Thus, the possibility that FHL2 regulates various innate and/or adaptive lymphocytes will be worth investigating.

It is noteworthy that FHL2 deficiency does not fully abrogate iNKT1 differentiation suggesting that commitment toward iNKT1 lineage may be under several and potentially redundant layers of regulation. Accordingly, a study elegantly demonstrated that PLZF silencing during iNKT1 commitment was under the control of a family of miRNAs (Let-7) (Pobezinsky et al., 2015).

Our data also indicate that multiple subsets of iNKT cells can naturally egress the thymus including both iNKT precursors (iNKT2a, iNKT2b, iNKT1a) and fully differentiated subsets (iNKT1c and iNKT17). The capacity of iNKT1 cells to leave the thymus is controversial since they have been reported as longterm thymic residents (Berzins et al., 2006; Crawford et al., 2013; Drennan et al., 2009), although a pioneer study revealed the presence of some iNKT1 cells in recent thymic emigrants (Pellicci et al., 2002). In line with this latter study, our results pinpoint iNKT1c as potential thymic emigrants despite the majority of iNKT1 (i.e., iNKT1b) appears retained in the thymus where their precise functions in tissue homeostasis remain unknown.

This may provide a strong advantage for the host in response to a peripheral insult such as infection by having on-site both iNKT subsets that can readily respond as well as iNKT precursors that can differentiate on-demand according to the inflammatory context.

Other functional iNKT subsets have been identified in the periphery such as iNKT10 (Sag et al., 2014) and iNKT $_{\mathrm{FH}}$ (Chang et al., 2011). However, we did not evidence such subsets based on our scRNA-seq analysis that mainly supports extrathymic requirements. In agreement, a recent study demonstrated that longterm activation was required to observe the emergence of such functions irrespective of the subset (Cameron and Godfrey, 2018).

Altogether, we propose a model for the development of effector iNKT cells. Our single-cell approach highlights the dynamic sequence of transcriptional events that illustrates how iNKT cell can reach such a wide variety of specificities. Thus, some of the genes described here and modulated during this developmental pathway will likely constitute candidate molecules in iNKT cell biology with potential impact in the context of infection, cancer, and inflammation.

\section{STAR $\star$ METHODS}

Detailed methods are provided in the online version of this paper and include the following:

- KEY RESOURCES TABLE

- RESOURCE AVAILABILITY
O Lead Contact

- Materials Availability

O Data and Code Availability

- EXPERIMENTAL MODEL AND SUBJECT DETAILS $\bigcirc$ Mice

- METHOD DETAILS

O Tissue preparation and flow cytometry

O Single-cell RNA-seq and data pre-processing

O Single-cell RNA-seq analysis

O Statistical analysis and visualization

O Cell cycle Analysis

O Data integration

- Trajectory inference using PAGA

- Functional enrichment

O Artificial thymic retention

O Reaggregate Thymus Organ Cultures (RTOC)

- QUANTIFICATION AND STATISTICAL ANALYSIS

\section{SUPPLEMENTAL INFORMATION}

Supplemental Information can be found online at https://doi.org/10.1016/j. celrep.2020.108116.

\section{ACKNOWLEDGMENTS}

This work was supported by the Agence Nationale de la Recherche "JCJC program" (ANR-19-CE15-0032-01) and by the Region Centre-Val de Loire under the program "ARD 2020 Biomédicaments" (Project 7UP). M.S.-T. and C.P. are supported by Inserm. F.T. and M.L.-d.-M. are supported by CNRS. T.B., L.G., and C.B. are supported by the University of Tours. Roxane Lemoine (BCR Facility, University of Tours) and Julie Cazareth (CNRS, IPMC, SophiaAntipolis) are acknowledged for their assistance with cell sorting. We acknowledge the UCAGenomiX platform, partner of the National Infrastructure France Génomique, supported by the Commissariat Aux Grands Investissements (ANR-10-INBS-09-03, ANR-10-INBS-09-02). Y. Wei (Institut Pasteur), M. Kronenberg (La Jolla Institute for Allergy \& Immunology), J. Van Meerwijk (CPTP, Toulouse), and P. Fink (University of Washington) are acknowledged for the kind gift of $F h / 2^{-/-}$, Traj $18^{-/-}$, and Rag2 ${ }^{\text {GFP }}$ mice, respectively. We also thank the University of Tours animal facility for excellent mouse husbandry and care. We thank the NIH tetramer core facility (Emory University) for providing CD1d tetramers. The graphical abstract was created using BioRender.com.

\section{AUTHOR CONTRIBUTIONS}

K.L. designed and performed the bioinformatic analysis and analyzed data. C.d.A.H., G.B., C.D., L.G., C.B., V.M., and Y.J. performed the experiments. F.T., M.S., M.L.-d.-M., and T.M. provided key materials and critical input. T.B. and C.P. supervised the project, performed experiments, analyzed data, and wrote the paper with the input of all authors.

\section{DECLARATION OF INTERESTS}

The authors declare no competing interests.

Received: May 12, 2020

Revised: July 24, 2020

Accepted: August 17, 2020

Published: September 8, 2020

\section{REFERENCES}

Aliahmad, P., and Kaye, J. (2008). Development of all CD4 T lineages requires nuclear factor TOX. J. Exp. Med. 205, 245-256. 


\section{CellPress}

Baranek, T., Morello, E., Valayer, A., Aimar, R.-F., Bréa, D., Henry, C., Besnard, A.-G., Dalloneau, E., Guillon, A., Dequin, P.-F., et al. (2017). FHL2 Regulates Natural Killer Cell Development and Activation during Streptococcus pneumoniae Infection. Front. Immunol. 8, 123.

Bendelac, A., Savage, P.B., and Teyton, L. (2007). The biology of NKT cells. Annu. Rev. Immunol. 25, 297-336.

Benlagha, K., Kyin, T., Beavis, A., Teyton, L., and Bendelac, A. (2002). A thymic precursor to the NK T cell lineage. Science 296, 553-555.

Benlagha, K., Wei, D.G., Veiga, J., Teyton, L., and Bendelac, A. (2005). Characterization of the early stages of thymic NKT cell development. J. Exp. Med. 202, 485-492.

Berzins, S.P., McNab, F.W., Jones, C.M., Smyth, M.J., and Godfrey, D.I. (2006). Long-term retention of mature NK1.1+ NKT cells in the thymus. J. Immunol. 176, 4059-4065.

Boursalian, T.E., Golob, J., Soper, D.M., Cooper, C.J., and Fink, P.J. (2004). Continued maturation of thymic emigrants in the periphery. Nat. Immunol. 5, 418-425.

Cameron, G., and Godfrey, D.I. (2018). Differential surface phenotype and context-dependent reactivity of functionally diverse NKT cells. Immunol. Cell Biol. Published online March 5, 2018. https://doi.org/10.1111/imcb.12034.

Carr, T., Krishnamoorthy, V., Yu, S., Xue, H.-H., Kee, B.L., and Verykokakis, M. (2015). The transcription factor lymphoid enhancer factor 1 controls invariant natural killer T cell expansion and Th2-type effector differentiation. J. Exp. Med. 212, 793-807.

Chandra, S., Zhao, M., Budelsky, A., de Mingo Pulido, A., Day, J., Fu, Z., Siegel, L., Smith, D., and Kronenberg, M. (2015). A new mouse strain for the analysis of invariant NKT cell function. Nat. Immunol. 16, 799-800.

Chang, P.-P., Barral, P., Fitch, J., Pratama, A., Ma, C.S., Kallies, A., Hogan, J.J., Cerundolo, V., Tangye, S.G., Bittman, R., et al. (2011). Identification of Bcl-6-dependent follicular helper NKT cells that provide cognate help for B cell responses. Nat. Immunol. 13, 35-43.

Chu, P.H., Bardwell, W.M., Gu, Y., Ross, J., Jr., and Chen, J. (2000). FHL2 (SLIM3) is not essential for cardiac development and function. Mol. Cell. Biol. 20, 7460-7462.

Crawford, G., Enders, A., Gileadi, U., Stankovic, S., Zhang, Q., Lambe, T., Crockford, T.L., Lockstone, H.E., Freeman, A., Arkwright, P.D., et al. (2013). DOCK8 is critical for the survival and function of NKT cells. Blood 122, 2052-2061.

Cruz Tleugabulova, M., Zhao, M., Lau, I., Kuypers, M., Wirianto, C., Umaña, J.M., Lin, Q., Kronenberg, M., and Mallevaey, T. (2019). The Protein Phosphatase Shp1 Regulates Invariant NKT Cell Effector Differentiation Independently of TCR and Slam Signaling. J. Immunol. 202, 2276-2286.

Drennan, M.B., Franki, A.-S., Dewint, P., Van Beneden, K., Seeuws, S., van de Pavert, S.A., Reilly, E.C., Verbruggen, G., Lane, T.E., Mebius, R.E., et al. (2009). Cutting edge: the chemokine receptor CXCR3 retains invariant NK T cells in the thymus. J. Immunol. 183, 2213-2216.

Engel, I., Seumois, G., Chavez, L., Samaniego-Castruita, D., White, B., Chawla, A., Mock, D., Vijayanand, P., and Kronenberg, M. (2016). Innate-like functions of natural killer T cell subsets result from highly divergent gene programs. Nat. Immunol. 17, 728-739.

Filén, S., and Lahesmaa, R. (2010). GIMAP Proteins in T-Lymphocytes. J. Signal Transduct. 2010, 268589.

Georgiev, H., Ravens, I., Benarafa, C., Förster, R., and Bernhardt, G. (2016). Distinct gene expression patterns correlate with developmental and functional traits of iNKT subsets. Nat. Commun. 7, 13116.

Godfrey, D.I., Stankovic, S., and Baxter, A.G. (2010). Raising the NKT cell family. Nat. Immunol. 11, 197-206.

Henao-Mejia, J., Williams, A., Goff, L.A., Staron, M., Licona-Limón, P., Kaech, S.M., Nakayama, M., Rinn, J.L., and Flavell, R.A. (2013). The microRNA miR181 is a critical cellular metabolic rheostat essential for NKT cell ontogenesis and lymphocyte development and homeostasis. Immunity 38, 984-997.

Kimura, M.Y., Igi, A., Hayashizaki, K., Mita, Y., Shinzawa, M., Kadakia, T., Endo, Y., Ogawa, S., Yagi, R., Motohashi, S., et al. (2018). CD69 prevents
$\mathrm{PLZF}^{\mathrm{hi}}$ innate precursors from prematurely exiting the thymus and aborting NKT2 cell differentiation. Nat. Commun. 9, 3749.

Kiselev, V.Y., Kirschner, K., Schaub, M.T., Andrews, T., Yiu, A., Chandra, T., Natarajan, K.N., Reik, W., Barahona, M., Green, A.R., and Hemberg, M. (2017). SC3: consensus clustering of single-cell RNA-seq data. Nat. Methods $14,483-486$.

Koay, H.-F., Gherardin, N.A., Enders, A., Loh, L., Mackay, L.K., Almeida, C.F., Russ, B.E., Nold-Petry, C.A., Nold, M.F., Bedoui, S., et al. (2016). A three-stage intrathymic development pathway for the mucosal-associated invariant T cell lineage. Nat. Immunol. 17, 1300-1311.

Koay, H.-F., Su, S., Amann-Zalcenstein, D., Daley, S.R., Comerford, I., Miosge, L., Whyte, C.E., Konstantinov, I.E., d'Udekem, Y., Baldwin, T., et al. (2019). A divergent transcriptional landscape underpins the development and functional branching of MAIT cells. Sci. Immunol. 4, eaay6039.

Kroetz, D.N., and Deepe, G.S., Jr. (2011). An aberrant thymus in CCR5-/- mice is coupled with an enhanced adaptive immune response in fungal infection. J. Immunol. 186, 5949-5955.

Lee, Y., Ahn, C., Han, J., Choi, H., Kim, J., Yim, J., Lee, J., Provost, P., Rådmark, O., Kim, S., and Kim, V.N. (2003). The nuclear RNase III Drosha initiates microRNA processing. Nature 425, 415-419.

Lee, Y.J., Holzapfel, K.L., Zhu, J., Jameson, S.C., and Hogquist, K.A. (2013). Steady-state production of IL-4 modulates immunity in mouse strains and is determined by lineage diversity of iNKT cells. Nat. Immunol. 14, 1146-1154.

Lee, Y.J., Starrett, G.J., Lee, S.T., Yang, R., Henzler, C.M., Jameson, S.C., and Hogquist, K.A. (2016). Lineage-Specific Effector Signatures of Invariant NKT Cells Are Shared amongst $\gamma \delta$ T, Innate Lymphoid, and Th Cells. J. Immunol. 197, 1460-1470.

Legoux, F., Gilet, J., Procopio, E., Echasserieau, K., Bernardeau, K., and Lantz, O. (2019). Molecular mechanisms of lineage decisions in metabolitespecific T cells. Nat. Immunol. 20, 1244-1255.

Li, J., Wu, D., Jiang, N., and Zhuang, Y. (2013). Combined deletion of Id2 and Id3 genes reveals multiple roles for E proteins in invariant NKT cell development and expansion. J. Immunol. 191, 5052-5064.

Lu, Y., Zhong, M.-C., Qian, J., Calderon, V., Cruz Tleugabulova, M., Mallevaey, T., and Veillette, A. (2019). SLAM receptors foster iNKT cell development by reducing TCR signal strength after positive selection. Nat. Immunol. 20, 447-457.

Malhotra, N., Qi, Y., Spidale, N.A., Frascoli, M., Miu, B., Cho, O., Sylvia, K., and Kang, J. (2018). SOX4 controls invariant NKT cell differentiation by tuning TCR signaling. J. Exp. Med. 215, 2887-2900.

Mandala, S., Hajdu, R., Bergstrom, J., Quackenbush, E., Xie, J., Milligan, J., Thornton, R., Shei, G.-J., Card, D., Keohane, C., et al. (2002). Alteration of lymphocyte trafficking by sphingosine-1-phosphate receptor agonists. Science 296, 346-349.

Matsuda, J.L., Gapin, L., Sidobre, S., Kieper, W.C., Tan, J.T., Ceredig, R., Surh, C.D., and Kronenberg, M. (2002). Homeostasis of V alpha 14i NKT cells. Nat. Immunol. 3, 966-974.

Milpied, P., Massot, B., Renand, A., Diem, S., Herbelin, A., Leite-de-Moraes, M., Rubio, M.-T., and Hermine, O. (2011). IL-17-producing invariant NKT cells in lymphoid organs are recent thymic emigrants identified by neuropilin-1 expression. Blood 118, 2993-3002.

Moran, A.E., Holzapfel, K.L., Xing, Y., Cunningham, N.R., Maltzman, J.S., Punt, J., and Hogquist, K.A. (2011). T cell receptor signal strength in Treg and iNKT cell development demonstrated by a novel fluorescent reporter mouse. J. Exp. Med. 208, 1279-1289.

Müller, U., Steinhoff, U., Reis, L.F., Hemmi, S., Pavlovic, J., Zinkernagel, R.M., and Aguet, M. (1994). Functional role of type I and type II interferons in antiviral defense. Science 264, 1918-1921.

Neil, S.J.D., Zang, T., and Bieniasz, P.D. (2008). Tetherin inhibits retrovirus release and is antagonized by HIV-1 Vpu. Nature 451, 425-430.

Paget, C., and Trottein, F. (2013). Role of type 1 natural killer T cells in pulmonary immunity. Mucosal Immunol. 6, 1054-1067. 


\section{Cell Reports}

Resource

Paget, C., Mallevaey, T., Speak, A.O., Torres, D., Fontaine, J., Sheehan, K.C.F., Capron, M., Ryffel, B., Faveeuw, C., Leite de Moraes, M., et al. (2007). Activation of invariant NKT cells by toll-like receptor 9-stimulated dendritic cells requires type I interferon and charged glycosphingolipids. Immunity 27, 597-609.

Pellicci, D.G., Hammond, K.J.L., Uldrich, A.P., Baxter, A.G., Smyth, M.J., and Godfrey, D.I. (2002). A natural killer T (NKT) cell developmental pathway ilnvolving a thymus-dependent NK1.1(-)CD4(+) CD1d-dependent precursor stage. J. Exp. Med. 195, 835-844.

Pobezinsky, L.A., Etzensperger, R., Jeurling, S., Alag, A., Kadakia, T., McCaughtry, T.M., Kimura, M.Y., Sharrow, S.O., Guinter, T.I., Feigenbaum, L., and Singer, A. (2015). Let-7 microRNAs target the lineage-specific transcription factor PLZF to regulate terminal NKT cell differentiation and effector function. Nat. Immunol. 16, 517-524.

Sag, D., Krause, P., Hedrick, C.C., Kronenberg, M., and Wingender, G. (2014). IL-10-producing NKT10 cells are a distinct regulatory invariant NKT cell subset. J. Clin. Invest. 124, 3725-3740.

Terashima, A., Watarai, H., Inoue, S., Sekine, E., Nakagawa, R., Hase, K., Iwamura, C., Nakajima, H., Nakayama, T., and Taniguchi, M. (2008). A novel subset of mouse NKT cells bearing the IL-17 receptor B responds to IL-25 and contributes to airway hyperreactivity. J. Exp. Med. 205, 2727-2733.
Tran, M.K., Kurakula, K., Koenis, D.S., and de Vries, C.J.M. (2016). Proteinprotein interactions of the LIM-only protein FHL2 and functional implication of the interactions relevant in cardiovascular disease. Biochim. Biophys. Acta 1863, 219-228.

Uehara, S., Grinberg, A., Farber, J.M., and Love, P.E. (2002). A role for CCR9 in T lymphocyte development and migration. J. Immunol. 168, 2811-2819.

Wang, H., and Hogquist, K.A. (2018). CCR7 defines a precursor for murine iNKT cells in thymus and periphery. eLife 7, e34793.

Watarai, H., Sekine-Kondo, E., Shigeura, T., Motomura, Y., Yasuda, T., Satoh, R., Yoshida, H., Kubo, M., Kawamoto, H., Koseki, H., and Taniguchi, M. (2012) Development and function of invariant natural killer T cells producing $T(h) 2-$ and T(h)17-cytokines. PLoS Biol. 10, e1001255.

Weinreich, M.A., and Hogquist, K.A. (2008). Thymic emigration: when and how T cells leave home. J. Immunol. 181, 2265-2270.

Wolf, F.A., Angerer, P., and Theis, F.J. (2018). SCANPY: large-scale single-cell gene expression data analysis. Genome Biol. 19, 15.

Wolf, F.A., Hamey, F.K., Plass, M., Solana, J., Dahlin, J.S., Göttgens, B., Rajewsky, N., Simon, L., and Theis, F.J. (2019). PAGA: graph abstraction reconciles clustering with trajectory inference through a topology preserving map of single cells. Genome Biol. 20, 59. 


\section{STAR $\star$ METHODS}

\section{KEY RESOURCES TABLE}

\begin{tabular}{|c|c|c|}
\hline REAGENT or RESOURCE & SOURCE & IDENTIFIER \\
\hline \multicolumn{3}{|l|}{ Antibodies and CD1d tetramers } \\
\hline Unloaded CD1d tetramer PE & $\begin{array}{l}\text { NIH Tetramer core facility, Emory } \\
\text { University, Atlanta, GA }\end{array}$ & N/A \\
\hline Unloaded CD1d tetramer Brilliant Violet 421 & NIH Tetramer core facility & $\mathrm{N} / \mathrm{A}$ \\
\hline PBS57 CD1d tetramer PE & NIH Tetramer core facility & N/A \\
\hline PBS57 CD1d tetramer Brilliant Violet 421 & NIH Tetramer core facility & N/A \\
\hline CD45 (30-F11) APC-eFluor780 & eBioscience, San Diego, CA, USA & RRID:AB_1548781 \\
\hline CD24 (M1/69) Alexa Fluor 488 & BioLegend, San Diego, CA, USA & RRID:AB_493482 \\
\hline CD24 (M1/69) APC-eFluor780 & eBioscience & RRID:AB_10853172 \\
\hline NK1.1 (PK136) Brilliant Blue 700 & BD Biosciences, San Diego, CA, USA & RRID:AB_2744491 \\
\hline CD3 (145-2C11) Alexa Fluor 488 & BioLegend & RRID:AB_389300 \\
\hline CD3 (145-2C11) PE-Cy7 & BioLegend & RRID:AB_312685 \\
\hline TCR $\beta(H 57-597)$ APC & BioLegend & RRID:AB_313435 \\
\hline TCR $\beta$ (H57-597) Alexa Fluor 700 & BioLegend & RRID:AB_1027648 \\
\hline CD44 (IM7) PE-Cy7 & BioLegend & RRID:AB_830787 \\
\hline CD138 (281-2) PE & BioLegend & RRID:AB_10916119 \\
\hline CD138 (281-2) Brilliant Violet 421 & BioLegend & RRID:AB_2565621 \\
\hline CD138 (281-2) PE-Cy7 & BioLegend & RRID:AB_2562198 \\
\hline Sca-1 (D7) APC & BioLegend & RRID:AB_313349 \\
\hline Sca-1 (D7) Super Bright 600 & eBioscience & RRID:AB_2663622 \\
\hline Sca-1 (D7) PerCP-Cy5.5 & BioLegend & RRID:AB_893615 \\
\hline FR4 (12A5) Brilliant Violet 786 & BD Biosciences & RRID:AB_2742014 \\
\hline FR4 (12A5) APC-Fire 750 & BioLegend & RRID:AB_2721485 \\
\hline CD19 (1D3) Super Bright 702 & eBioscience & RRID:AB_2717135 \\
\hline CD4 (GK1.5) APC/Fire750 & BioLegend & RRID:AB_2572111 \\
\hline IFN- $\gamma$ (XMG1.2) Pacific Blue & BioLegend & RRID:AB_893526 \\
\hline IFN- $\gamma$ (XMG1.2) APC & BioLegend & RRID:AB_315404 \\
\hline IL-4 (11B11) PE & BioLegend & RRID:AB_315318 \\
\hline IL-17A (TC11-18H10) Brilliant Violet 650 & BioLegend & RRID:AB_2686975 \\
\hline Ki67 (11F6) FITC & BioLegend & RRID:AB_2814055 \\
\hline ROR $\gamma$ t (Q31-378) PE-CF594 & BD Biosciences & RRID:AB_2651150 \\
\hline ROR $\gamma t$ (Q31-378) Brilliant Violet 421 & BD Biosciences & RRID:AB_2687545 \\
\hline PLZF (R17-809) Alexa Fluor 647 & BD Biosciences & RRID:AB_2738238 \\
\hline T-bet (4B10) PE-Cy7 & BioLegend & RRID:AB_2561761 \\
\hline T-bet (4B10) PE & BioLegend & RRID:AB_2200542 \\
\hline \multicolumn{3}{|c|}{ Chemicals, Medium, kit reagents and recombinant proteins } \\
\hline LIVE/DEAD Fixable Aqua Dead Cell Stain kit & ThermoFisher Scientific, Illkirch, France & L34957 \\
\hline MitoProbe TMRM & ThermoFisher Scientific & M20036 \\
\hline MitoTracker Deep red & ThermoFisher Scientific & M22426 \\
\hline Phorbol 12-myristate 13-acetate & $\begin{array}{l}\text { Sigma-Aldrich, Saint Quantin Fallavier, } \\
\text { France }\end{array}$ & P8139 \\
\hline Ionomycin & Sigma-Aldrich & 10634 \\
\hline Fixation/Permeabilization Solution Kit & BD Biosciences & $\# 554714$ \\
\hline
\end{tabular}




\begin{tabular}{|c|c|c|}
\hline \multicolumn{3}{|l|}{ Continued } \\
\hline REAGENT or RESOURCE & SOURCE & IDENTIFIER \\
\hline $\begin{array}{l}\text { True-Nuclear Transcription Factor Buffer } \\
\text { Set }\end{array}$ & BioLegend & \#424401 \\
\hline Anti-PE microbeads Mojo Sort & BioLegend & \#480080 \\
\hline RPMI 1640 & ThermoFisher Sientific & $\# 61870150$ \\
\hline Basal DMEM Medium, No HEPES & ThermoFisher Sientific & \#A1443001 \\
\hline Red blood lysis buffer & Sigma-Aldrich & $\mathrm{R} 7757$ \\
\hline Brefeldin A & eBioscience & $\# 00-4506-51$ \\
\hline Monensin & eBioscience & $\# 00-4505-51$ \\
\hline NextSeq 500 High Output v2 kit & Illumina Inc., San Diego, CA, USA & N/A \\
\hline FTY720 & Sigma-Aldrich & SML0700 \\
\hline rhlL-7 & Miltenyi-Biotec, Paris, France & $\# 130-095-362$ \\
\hline rmlL-15 & Miltenyi-Biotec & $\# 130-094-071$ \\
\hline Ficoll-Paque PLUS & Sigma-Aldrich & GE17-1440-02 \\
\hline \multicolumn{3}{|l|}{ Deposited Data } \\
\hline $\begin{array}{l}\text { Raw data sc RNA-Seq of } F h l 2^{+/+} \text {and } \\
\text { Fhl2 }{ }^{-/-} \text {iNKT thymocytes }\end{array}$ & This study & GEO: GSE141895 \\
\hline \multicolumn{3}{|l|}{ Experimental Models: Organisms/Strains } \\
\hline C57BL/6J (B6) & Janvier Labs, Le Genest-St-Isle, France & N/A \\
\hline $\mathrm{B} 6 \mathrm{Fh} / 2^{-1-}$ & (Chu et al., 2000) & N/A \\
\hline B6 Ifnar1 ${ }^{-1-}$ & (Müller et al., 1994) & $\mathrm{N} / \mathrm{A}$ \\
\hline B6 Traj18 ${ }^{-1-}$ & (Chandra et al., 2015) & $\mathrm{N} / \mathrm{A}$ \\
\hline B6 Rag2 ${ }^{\text {GFP }}$ & (Boursalian et al., 2004; Moran et al., 2011) & $\mathrm{N} / \mathrm{A}$ \\
\hline \multicolumn{3}{|l|}{ Materials, Software and Algorithms } \\
\hline Prism 8.0 & Graphpad software & $\mathrm{N} / \mathrm{A}$ \\
\hline VenturiOne & Applied Cytometry, Sheffield, UK & $\mathrm{N} / \mathrm{A}$ \\
\hline FlowJo v10 & BD Biosciences & $\mathrm{N} / \mathrm{A}$ \\
\hline LSR Fortessa & BD Biosciences & $\mathrm{N} / \mathrm{A}$ \\
\hline BD FACSMelody & BD Biosciences & $\mathrm{N} / \mathrm{A}$ \\
\hline BD FACSAria III & BD Biosciences & N/A \\
\hline MACS Quant & Miltenyi Biotec & N/A \\
\hline Chromium $3^{\prime}$ chip & 10X Genomics, Pleasanton, CA, USA & N/A \\
\hline NextSeq500 Illumina & Illumina Inc. & $\mathrm{N} / \mathrm{A}$ \\
\hline Python & $\begin{array}{l}\text { Python Software Foundation, Wilmington, } \\
\text { DE, USA }\end{array}$ & $\mathrm{N} / \mathrm{A}$ \\
\hline Scanpy & (Wolf et al., 2018) & NA \\
\hline PAGA & (Wolf et al., 2019) & NA \\
\hline
\end{tabular}

\section{RESOURCE AVAILABILITY}

\section{Lead Contact}

Further information and requests for resources and reagents should be directed to the Lead Contact, Christophe Paget (christophe. paget@inserm.fr).

\section{Materials Availability}

This study did not generate new unique reagents.

\section{Data and Code Availability}

The accession number for the sc RNA-seq of WT and Fh/2 ${ }^{-/-}$thymic iNKT cells reported in this paper is GSE141895. Data are also available through a dedicated web interface (https://www.genomique.eu/cellbrowser/ucagenomix/baranek2020/). All Python and R scripts used for single cell statistical analysis can be accessed on GitHub (https://github.com/ucagenomix/sc.baranek.2020). 


\section{EXPERIMENTAL MODEL AND SUBJECT DETAILS}

\section{Mice}

Wild-type (WT) C57BL/6JRj (B6) mice were purchased from Janvier Labs. B6 Fh/2 ${ }^{-/-}$, Ifnar1 ${ }^{-/-}$, Traj18 ${ }^{-/-}$and Rag2 ${ }^{\text {GFP }}$ mice have been previously described (Boursalian et al., 2004; Chu et al., 2000; Moran et al., 2011; Müller et al., 1994; Chandra et al., 2015). Experiments using $F h / 2^{-1-}$ mice were confirmed using $F h / 2^{-/-}$and $F h / 2^{+/+}$littermates obtained from intercrossing of $F h / 2^{+/-}$mice. Mice were bred and maintained under specific pathogen-free conditions in-house and were used at 5-8 weeks of age. All animal experimentation was performed according to the national governmental guidelines and was approved by our local and national ethics committee (CEEA.19, \#201604071220401-4885).

\section{METHOD DETAILS}

Tissue preparation and flow cytometry

Single-cell suspensions were prepared from thymus, liver, and spleen as previously described (Paget et al., 2007). Red blood cells were removed using a red blood cell lysis buffer. In some cases, cells were stimulated for 4 hours in complete media containing PMA $(100 \mathrm{ng} / \mathrm{ml})$ and ionomycin $(1 \mu \mathrm{g} / \mathrm{ml})$ in presence of protein transport inhibitor cocktail added 30 min after stimulation. For intracellular staining, cells were first stained with antibodies to surface makers and viability dye (LIVE/DEAD Fixable Aqua Dead Cell Stain), then fixed and permeabilized using a Fixation/Permeabilization Solution Kit or a True-Nuclear Transcription Factor Buffer Set for intracytoplasmic intranuclear staining respectively. Events were acquired on either LSR Fortessa or a MACSQuant cytometers. Analyses were performed by using the VenturiOne software or FlowJo v10.

\section{Single-cell RNA-seq and data pre-processing}

Single cell suspension from 4 thymi were pooled and total iNKT thymocytes (CD45 ${ }^{+} \mathrm{CD}^{+}$PBS57-loaded CD1d tetramer $\left.{ }^{+}\right)$were sorted on an Aria III cell sorter (purity $>99 \%$ ) into non-HEPES medium containing $10 \%$ of FCS. Sorted cells were counted under a microscope and 10,000 cells were loaded onto a Chromium $3^{\prime}$ chip. Reverse transcription and library preparation were performed according to the manufacturer's protocol. Libraries were sequenced on a NextSeq 500 High Output v2 kit ( 75 cycles) that allows up to 91 cycles of paired-end sequencing: read 1 had a length of 26 bases that included the cell barcode and the UMl; read 2 had a length of 57 bases that contained the cDNA insert; Index reads for sample index of 8 bases. Cell Ranger Single-Cell Software Suite v2.0.0 was used to perform sample demultiplexing, barcode processing and single-cell $3^{\prime}$ gene counting using standards default parameters and Mus musculus build mm10.

\section{Single-cell RNA-seq analysis}

Cell and gene filtering

Raw gene expression matrices generated per sample were loaded and processed within Scanpy python workflow (Wolf et al., 2018). Cells having more than $5 \%$ of mitochondrial RNA were removed; then ribosomal, mitochondrial and genes expressed in less than 3 cells were removed.

Doublet removal

Unbiased identification of doublets (technical error) in samples was performed using Doublet Detection and Scrublet (https://github. com/JonathanShor/DoubletDetection, https://github.com/AllonKleinLab/scrublet). Using default parameters, we removed the cells predicted as doublets by any of the two methods.

Statistical analysis and visualization

Raw counts of remaining cells were normalized and log-transformed before highly variable genes (HVG) was identified using Scanpy HVG function (Parameters: min_mean $=0.0125$, max_mean $=3$, min_disp $=0.5$ ). Resulting HVG were summarized by principle component analysis, then the first 50 principle components were further summarized using UMAP dimensionality reduction. Cells were then clustered using the Louvain algorithm with a resolution of 1.5. Cell clusters in the resulting UMAP two-dimensional representation were annotated to known biological cell types using canonical marker genes identified using Scanpy rank_gene_group function with the Wilcoxon's rank test.

\section{Cell cycle Analysis}

Cell cycle scoring (S phase and G2M phase) was performed using Scanpy score_genes_cell_cycle function with cell cycle genes list from Tirosh et al. (https://science.sciencemag.org/content/352/6282/189).

\section{Data integration}

For $\mathrm{Fh} / 2^{+/+}$and $\mathrm{Fh} / 2^{-/-}$single cell transcriptome comparison, batch effect was removed using mutual nearest neighbors method implemented in 'mnn_correct' function in mnnpy python package (Haghverdi L) using intersection of individual sample highly variables gene sets. 
Trajectory inference using PAGA

For each of the two samples, cycling iNKT and NKT-ISG clusters was removed before independent trajectory inference analysis was performed using PAGA algorithm available within Scanpy.

Functional enrichment

Data gene sets were analyzed for functional enrichment with Enrichr (https://amp.pharm.mssm.edu/Enrichr/\#) using as the reference databases GO biological process 2018, GO molecular function 2018 and KEGG 2019 mouse.

Artificial thymic retention

Mice received daily $20 \mu \mathrm{g}$ of FTY720 intraperitoneally (i.p.) for seven days. Then thymi of treated mice were analyzed the day following the last administration.

\section{Reaggregate Thymus Organ Cultures (RTOC)}

Thymocytes were stained with PBS57-loaded CD1d tetramers and enriched using Mojo Sort. Then, iNKT subsets were sorted using a BD FACS Melody. $2 \times 10^{4}$ purified iNKT subsets (purity > 97\%; Figure S5) were cocultured with $2 \times 10^{7}$ of total thymocytes from Traj18 $8^{-/-}$mice in complete $10 \%$ FCS RPMI1640 containing recombinant IL-7 (20 ng/mL) and IL-15 (20 ng/mL). Cells were incubated for 4 days and dead cells were removed by Ficoll-Paque PLUS centrifugation prior analysis.

\section{QUANTIFICATION AND STATISTICAL ANALYSIS}

All data are presented as mean \pm SEM. All statistical analysis was performed by using GraphPad Prism software. The statistical significance was evaluated by using nonparametric unpaired Mann-Whitney $U$ tests in order to compare the means of biological replicates in each experimental group. Results with a $\mathrm{P}$ value of $<0.05$ were considered significant. ns: not significant; ${ }^{*} p<0.05$; ${ }^{\star *} p<$ $0.01 ;{ }^{* \star} p<0.001$.). 